\title{
AUTOMORPHISMS OF NEARLY FINITE COXETER GROUPS
}

\author{
W. N. FRANZSEN AND R. B. HOWLETT
}

\begin{abstract}
Suppose that $W$ is an infinite Coxeter group of finite rank $n$, and suppose that $W$ has a finite parabolic subgroup $W_{J}$ of rank $n-1$. Suppose also that the Coxeter diagram of $W$ has no edges with infinite labels. Then any automorphism of $W$ that preserves reflections lies in the subgroup of $\operatorname{Aut}(W)$ generated by the inner automorphisms and the automorphisms induced by symmetries of the Coxeter graph. If, in addition, $W_{J}$ is irreducible and every conjugacy class of reflections in $W$ has nonempty intersection with $W_{J}$, then all automorphisms of $W$ preserve reflections, and it follows that $\operatorname{Aut}(W)$ is the semi-direct product of $\operatorname{Inn}(W)$ by the group of graph automorphisms.
\end{abstract}

There is not much literature dealing with the automorphism groups of infinite Coxeter groups 1 It seems that complete results are known only for rank 3 Coxeter groups and the so-called right-angled Coxeter groups.

A Coxeter group is right-angled if the labels on all edges in the Coxeter diagram are $\infty$. These were investigated by James, [12], who described the automorphism groups of Coxeter groups whose diagrams have the following form:

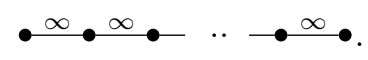

James's result was extended by Tits, [16], to include all irreducible right-angled Coxeter groups whose diagrams do not contain triangles. Finally, in [14], Mühlherr gave a presentation for the automorphism group of any right-angled Coxeter group.

The automorphism groups of infinite rank 3 Coxeter groups whose diagrams have no edges with infinite labels are described in [9]; in this case the automorphism group is the semi-direct product of $\operatorname{Inn}(W)$ and the group of graph automorphisms. The automorphism groups of rank 3 Coxeter groups with both finite and infinite edge labels are described in [7].

For the purposes of this paper, we say that an infinite Coxeter group is nearly finite if it has finite rank $n$ and has a finite parabolic subgroup of rank $n-1$. It is shown that if $W$ is nearly finite and does not have an edge labelled $\infty$ then the group of all automorphisms of $W$ that preserve reflections is the semi-direct product of $\operatorname{Inn}(W)$ and the group of graph automorphisms. In certain special cases we are able to show that all automorphisms of $W$ preserve reflections. In fact, if we restrict attention to infinite irreducible Coxeter groups whose diagrams have no infinite edge labels, then we know of no example having an automorphism that does not preserve reflections.

\section{PReliminaries}

Recall that a Coxeter group is a group with a presentation of the form

$$
\left.W=\operatorname{gp}\left\langle\left\{r_{a} \mid a \in \Pi\right\}\right|\left(r_{a} r_{b}\right)^{m_{a b}}=1 \text { for all } a, b \in \Pi\right\rangle
$$

where $\Pi$ is some indexing set, whose cardinality is called the rank of $W$, and the $m_{a b}$ satisfy the following conditions: $m_{a b}=m_{b a}$, each $m_{a b}$ lies in the set $\{m \in \mathbb{Z} \mid m \geq 1\} \cup\{\infty\}$, and $m_{a b}=1$ if and only if $a=b$. When $m_{a b}=\infty$ the relation $\left(r_{a} r_{b}\right)^{m_{a b}}=1$ is interpreted as vacuous. We shall restrict attention to finite rank groups with $m_{a b} \neq \infty$ for all $a, b \in \Pi$.

\footnotetext{
2000 Mathematics Subject Classification. Primary 20F55.

${ }^{1}$ The closely related question of whether a Coxeter group may contain more than one class of Coxeter generating sets is investigated in [5].
} 
As is well known, the isomorphism type of $W$ as an abstract group does not determine either the parameters $m_{a b}$ or the rank of $W$ as a Coxeter group. Hence we always assume that the presentation 1.1 is given; in particular, $\left\{r_{a} \mid a \in \Pi\right\}$ is a distinguished set of generators for the group $W$.

A reduced expression for an element $w \in W$ is a minimal length word expressing $w$ as a product of elements of the distinguished generating set. We define $l(w)$ to be the length of a reduced expression for $w$.

The Coxeter diagram of $W$ is a graph with vertex set $\Pi$ and edge set consisting of those pairs of vertices $\{a, b\}$ for which $m_{a b} \geq 3$. The edge $\{a, b\}$ is given the label $m_{a b}$. We say that $W$ is irreducible if its diagram is connected.

Let $\mathbb{R}$ be the real field, and $V$ the vector space over $\mathbb{R}$ with basis $\Pi$. Let $B$ the bilinear form on $V$ such that for all $a, b \in \Pi$,

$$
B(a, b)=-\cos \left(\pi / m_{a b}\right)
$$

To make our notation more compact we define $u \cdot v=B(u, v)$ for all $u, v \in V$. Note that $a \cdot a=1$ for all $a \in \Pi$, since $m_{a a}=1$.

For each $a \in V$ such that $a \cdot a=1$ the transformation of $V$ given by $v \mapsto v-2(a \cdot v) a$ is called the reflection along $a$. It is well known (see, for example, Corollary 5.4 of [11]) that $W$ has a faithful representation on $V$ such that, for all $a \in \Pi$, the element $r_{a}$ acts as the reflection along $a$. We shall identify elements of $W$ with their images in this representation. We also use the notation $r_{a}$ for the reflection along $a$ whenever $a \in V$ satisfies $a \cdot a=1$. It is straightforward to show that each reflection $r_{a}$ preserves the form $B$; hence all elements of $W$ preserve $B$. Furthermore, the equation $g r_{a} g^{-1}=r_{g a}$ holds for all $a \in V$ such that $a \cdot a=1$ and all transformations $g$ that preserve $B$.

We write $\operatorname{Ref}(W)$ for the set of all reflections in $W$. It is immediate from the above comments that if $\Phi=\{w a \mid w \in W, a \in \Pi\}$ then $\left\{r_{b} \mid b \in \Phi\right\} \subseteq \operatorname{Ref}(W)$.

The set $\Phi$ is called the root system of $W$, and elements of $\Phi$ are called roots. Elements of the basis $\Pi$ are called simple roots, and the reflections $r_{a}$ for $a \in \Pi$ are called simple reflections. A root is said to be positive if it has the form $\sum_{a \in \Pi} \lambda_{a} a$ with $\lambda_{a} \geq 0$ for all $a \in \Pi$, and negative otherwise. We write $\Phi^{+}$for the set of all positive roots and $\Phi^{-}$for the set of all negative roots.

Lemma 1. With the notation as above, the following statements hold.

(1) Every negative root has the form $\sum_{a \in \Pi} \lambda_{a} a$ with $\lambda_{a} \leq 0$ for all $a \in \Pi$. Furthermore, $\Phi^{-}=\left\{-b \mid b \in \Phi^{+}\right\}$.

(2) If $w \in W$ and $a \in \Pi$ then

$$
l\left(w r_{a}\right)= \begin{cases}l(w)+1 & \text { if } w a \in \Phi^{+} \\ l(w)-1 & \text { if } w a \in \Phi^{-}\end{cases}
$$

(3) If $t \in \operatorname{Ref}(W)$ then $t=r_{b}$ for some $b \in \Phi$.

(4) The group $W$ is finite if and only if the bilinear form $B$ is positive definite.

(5) The root system $\Phi$ is finite if and only if the group $W$ is finite.

Proof. Proofs of (1) and (2) can be found in [11, Section 5.4], Theorem 4.1 in [6] includes both (4) and (5), and (3) is [10, Lemma 2.2].

For each $w \in W$ we define $N(w)=\left\{b \in \Phi^{+} \mid w b \in \Phi^{-}\right\}$. By Part (2) of Lemma 1, if $w \neq 1$ then $N(w) \cap \Pi \neq \emptyset$. An easy induction shows that $N(w)$ has exactly $l(w)$ elements. In particular, $N(w)$ is a finite set. It is also easily shown that if $\Phi$ is finite then there is a unique $w \in W$ such that $N(w)=\Phi^{+}$. This element, which we denote by $w_{\Pi}$, is also the unique element of maximal length in $W$ (which is a finite group). 
We need the following simple fact.

Lemma 2. Suppose that $w \in W$ is an involution, and let $a \in N(w) \cap \Pi$. Then either $w a=-a$ or $l\left(r_{a} w r_{a}\right)=l(w)-2$.

Proof. Observe that $-w a \in \Phi^{+}$, since $a \in N(w)$. Now $N\left(r_{a}\right)=\{a\}$, since $a \in \Pi$, and so if $-w a \neq a$ it follows that $r_{a}(-w a) \in \Phi^{+}$. But this implies that $\left(r_{a} w\right) a \in \Phi^{-}$, and so by Lemma 1 combined with the obvious fact that each element has the same length as its inverse,

as claimed.

$$
l\left(r_{a} w r_{a}\right)=l\left(r_{a} w\right)-1=l\left(w r_{a}\right)-1=l(w)-2
$$

The following lemma is one of the key ingredients in the proof of our main theorem.

Lemma 3 (Brink [2]). Suppose that $b$ is a positive root, and write $b=\sum_{a \in \Pi} \lambda_{a} a$. For each $a \in \Pi$, if $\lambda_{a}>0$ then $\lambda_{a} \geq 1$.

\section{PARABOLIC SUBGROUPS AND REFLECTION PRESERVING AUTOMORPHISMS}

Let $W$ be a Coxeter group, and continue with the notation introduced in the preceding section. For each $I \subseteq \Pi$ we define $W_{I}$ to be the subgroup of $W$ generated by $\left\{r_{a} \mid a \in I\right\}$. These subgroups are called the standard parabolic subgroups of $W$. A parabolic subgroup of $W$ is any subgroup of the form $w W_{I} w^{-1}$ for some $w \in W$ and $I \subseteq \Pi$. We shall use the phrase "maximal parabolic subgroup" to mean "maximal proper parabolic subgroup".

It is clear that if $I \subseteq \Pi$ then $W_{I}$ preserves the subspace $V_{I}$ of $V$ spanned by $I$, and acts on this subspace as a Coxeter group with $I$ as its set of simple roots. We write $\Phi_{I}$ for the root system of $W_{I}$ in $V_{I}$, and $\Phi_{I}^{+}, \Phi_{I}^{-}$for the sets of positive and negative roots in $\Phi_{I}$.

Lemma 4. In the above situation, $\Phi_{I}=\Phi \cap V_{I}$.

Proof. For each $b=\sum_{a \in \Pi} \lambda_{a} a \in \Phi$ define $\operatorname{supp}(b)=\left\{a \in \Pi \mid \lambda_{a} \neq 0\right\}$. It is clear that if $b=w a$ for some $a \in I$ and $w \in W_{I}$ then $\operatorname{supp}(b) \subseteq I$; we must prove that the converse also holds. Without loss of generality we may assume that $b$ is positive.

Let $b \in \Phi^{+}$with $\operatorname{supp}(b) \subseteq I$. Since $r_{b} \neq 1$ we may choose a simple root $c \in N\left(r_{b}\right) \cap \Pi$. Then $c-2(b \cdot c) b=r_{b} c \in \Phi^{-}$, and so $b \cdot c>0$. Since $a \cdot c \leq 0$ for all $a \in \Pi \backslash\{c\}$ it follows that $c \in \operatorname{supp}(b)$.

We proceed by induction on $l\left(r_{b}\right)$. If $l\left(r_{b}\right)=1$ then we must have $b=c$, and $b=w a$ holds with $w=1 \in W_{I}$ and $a=c \in I$. Now suppose that $l\left(r_{b}\right)>1$, so that $b \neq c$, and put $d=r_{c} b$. Lemma 2 gives $l\left(r_{d}\right)=l\left(r_{c} r_{b} r_{c}\right)=l\left(r_{b}\right)-2$; moreover, since $d=b-2(c \cdot b) c$ we see that $\operatorname{supp}(d) \subseteq \operatorname{supp}(b)$. By the inductive hypothesis $d=w a$ for some $w \in W_{I}$ and $a \in I$, and since $c \in I$ it follows that $r_{c} w \in W_{I}$, and $b=\left(r_{c} w\right) a$ is an equation of the desired form.

The next proposition, classifying involutions in Coxeter groups, is a useful tool in the analysis of automorphisms.

Proposition 5 (Richardson [15]). Suppose that $w \in W$ is an involution. Then there is an $I \subseteq \Pi$ such that $W_{I}$ is finite, $w$ is conjugate to $w_{I}$ (the maximal length element of $W_{I}$ ) and $w_{I}$ is central in $W_{I}$.

Proof. Let $L=\{a \in \Pi \mid w a=-a\}$. First observe that $\Phi_{L}^{+} \subset N(w)$ is finite, and so, by Lemma 1. $W_{L}$ is finite. If $a \in L$ then $w r_{a} w=r_{w a}=r_{-a}=r_{a}$, and so it follows that $w$ centralizes $W_{L}$.

If $w=w_{L}$ then we are finished; so suppose that $w \neq w_{L}$. Then $w_{L} w \neq 1$, and so we may choose an $a \in N\left(w_{L} w\right) \cap \Pi$. If $w a \in \Phi^{+}$, then, as $w_{L} w a \in \Phi^{-}$, we have $w a \in N\left(w_{L}\right)=\Phi_{L}^{+}$. But then

$$
a=w(w a) \in w \Phi_{L}^{+}=\Phi_{L}^{-},
$$


which is a contradiction. Hence $a \in N(w) \cap \Pi$. Now $w a \neq-a$, since $w a=-a$ would mean that $a \in L$, and

$$
w_{L} w a=w_{L}(-a) \in w_{L} \Phi_{L}^{-}=\Phi_{L}^{+},
$$

contradicting $a \in N\left(w_{L} w\right)$. Hence $l\left(r_{a} w r_{a}\right)=l(w)-2$ by Lemma 2 , and we can use induction on the length to complete the proof.

Note that the above proof in fact shows that $w=t^{-1} w_{I} t$ for some $t \in W$ such that $l(w)=2 l(t)+l\left(w_{I}\right)$.

Our main tool in the analysis of automorphisms of infinite Coxeter groups is the following lemma, which appears in [1, Exercise 2d, p. 130].

Lemma 6 (Tits). If $W$ is a Coxeter group and $H \leq W$ is finite, then $H$ is contained in a finite parabolic subgroup of $W$.

One immediate consequence of Lemma 6 is that every maximal finite subgroup of a Coxeter group is parabolic.

Lemma 7 (Kilmoyer). Let $I, J \subseteq \Pi$. Then every $\left(W_{I}, W_{J}\right)$ double coset in $W$ contains a unique element of minimal length; moreover, if $d$ is the minimal length element of $W_{I} d W_{J}$ then $W_{I} \cap d W_{J} d^{-1}=W_{K}$, where $K=I \cap d J$.

Proof. See [4, Theorem 2.7.4].

Corollary 8. The intersection of a finite number of parabolic subgroups is a parabolic subgroup.

Proof. If $H$ and $K$ are parabolic subgroups then $H=x^{-1} W_{I} x$ and $K=y^{-1} W_{J} y$ for some $I, J \subseteq \Pi$ and $x, y \in W$. Let $d$ be the minimal length element in $W_{I} x y^{-1} W_{J}$, and choose $u \in W_{I}$ and $t \in W_{J}$ such that $d=u x y^{-1} t$. Then

$$
H \cap K=x^{-1} u^{-1} W_{I} u x \cap y^{-1} t W_{J} t^{-1} y=x^{-1} u^{-1}\left(W_{I} \cap d W_{J} d^{-1}\right) u x,
$$

which is a parabolic subgroup by Lemma 7 Induction completes the proof.

Since the image under any automorphism of a maximal finite subgroup must be another maximal finite subgroup, Corollary 8 immediately yields the following result.

Corollary 9. Let $W$ be an infinite Coxeter group, and $\alpha \in \operatorname{Aut}(W)$. If $H$ is a subgroup of $W$ that can be written as the intersection of a collection of maximal finite subgroups, then $\alpha(H)$ is a parabolic subgroup of $W$.

A special case of Corollary 9 provides a possible method for proving that an automorphism preserves reflections.

Corollary 10. If $W$ is an infinite Coxeter group, $\alpha \in \operatorname{Aut}(W)$ and $r$ is a reflection such that $\langle r\rangle$ can be written as an intersection of maximal finite subgroups, then $\alpha(r)$ is a reflection.

Suppose that $I \subseteq \Pi$ is such that $W_{I}$ is a maximal finite standard parabolic subgroup of $W$ (in the sense that $W_{I}$ is finite and $W_{J}$ is infinite for all $J$ with $I \varsubsetneqq J \subseteq \Pi$ ). We shall show that $W_{I}$ is not properly contained in any finite subgroup of $W$.

Lemma 11. Let $W_{I}$ be a maximal finite standard parabolic subgroup. Then $W_{I}$ is not conjugate to a subgroup of any other finite standard parabolic subgroup.

Proof. Suppose that $W_{I} \subseteq t W_{K} t^{-1}$ for some $t \in W$ and some $K \subseteq \Pi$ such that $W_{K}$ is finite and $K \neq I$. These assumptions are not altered by replacing $t$ by another element of the double coset $W_{I} t W_{K}$; so we may assume that $t$ is the minimal length element of $W_{I} t W_{K}$. By Corollary 7 it follows that $W_{I}=W_{I \cap t K}$, and so $I \subseteq t K$.

Since $W_{I}$ is a maximal finite standard parabolic subgroup, $t \neq 1$. So, by Lemma 1, we may choose a simple root $c$ such that $t^{-1} c=d$ is negative. As $t$ has minimal length in $t W_{K}$, Lemma 1 guarantees that $t a$ is positive for all $a \in K$, and hence $t b$ is positive for all $b \in \Phi_{K}^{+}$. 
But $-d$ is positive while $t(-d)=-c$ is negative, and so we conclude that $d$ is not in $\Phi_{K}$. Thus when $d=t^{-1} c$ is expressed as a linear combination of simple roots, some $e \notin K$ appears with a negative coefficient. Now suppose that $b \in \Phi_{I \cup\{c\}}^{+} \backslash \Phi_{I}$, so that $b=\lambda c+v$ for some $\lambda>0$ and some $v \in V_{I}$. Since $t^{-1} I \subseteq K$ it follows that $t^{-1} v \in V_{K}$, and hence $t^{-1} b=\lambda\left(t^{-1} c\right)+t^{-1} v$ involves $e$ with negative coefficient. So $t^{-1} b \in \Phi^{-}$. But $\Phi_{I \cup\{c\}}$ is infinite, while $\Phi_{I}$ is not. So $t^{-1}$ takes an infinite number of positive roots to negative roots, and hence has infinite length. This is a contradiction.

Corollary 12. If $W$ is any infinite Coxeter group, then all maximal finite standard parabolic subgroups of $W$ are maximal finite subgroups of $W$.

Proof. If $W_{I}$ is a maximal finite standard parabolic subgroup but not a maximal finite subgroup then $W_{I} \leq t W_{J} t^{-1}$ for some $t \in W$ and $J \subseteq \Pi$ with $\left|W_{I}\right|<\left|W_{J}\right|<\infty$, by Lemma 6 But this contradicts Lemma 11

If $I$ and $J$ are disjoint subsets of $\Pi$ such that $m_{a b}=2$ for all $a \in I$ and $b \in J$, then $V_{I}$ and $V_{J}$ are orthogonal to each other, and it follows readily that $W_{I \cup J}=W_{I} \times W_{J}$. Moreover, $\Phi_{I \cup J}=W_{I \cup J}(I \cup J)=W_{I} I \cup W_{J} J=\Phi_{I} \cup \Phi_{J}$, since each $w \in W_{I}$ fixes each $a \in J$ and each $w \in W_{J}$ fixes each $a \in I$. So we obtain the following result.

Lemma 13. Let $W$ be a Coxeter group of rank $n$ and $\Pi$ the set of simple roots. If I and $J$ are disjoint subsets of $\Pi$ such that no edge of the Coxeter diagram joins a root in I and a root in $J$, then

$$
\operatorname{Ref}\left(W_{I \cup J}\right)=\operatorname{Ref}\left(W_{I}\right) \dot{\cup} \operatorname{Ref}\left(W_{J}\right) .
$$

(where the symbol $\cup$ signifies a disjoint union).

Corresponding to the connected components of the Coxeter diagram we obtain a decomposition $\Pi=L_{1} \dot{\cup} L_{2} \dot{\cup} \ldots \dot{\cup} L_{m}$ such $a, b \in \Pi$ lie in the same subset $L_{i}$ if and only if there exists a chain of simple roots $a=a_{1}, a_{2}, \ldots, a_{k}=b$ such that the reflections along consecutive terms do not commute. We call the $L_{i}$ the irreducible components of $\Pi$, and the corresponding standard parabolic subgroups $W_{L_{i}}$ the irreducible components of $W$. Note that $W=W_{L_{1}} \times W_{L_{2}} \times \cdots \times W_{L_{m}}$ and $\operatorname{Ref}(W)=\operatorname{Ref}\left(W_{L_{1}}\right) \cup \cdots \dot{\operatorname{Ref}}\left(W_{L_{m}}\right)$.

It is clear that reflections belonging to different irreducible components commute. On the other hand if $b \in \Phi$ is not simple then it is clear that there exists a simple root $a$ such that $r_{a}(b) \neq b$, and so $r_{a}$ and $r_{b}$ do not commute. It follows that reflections $r$ and $r^{\prime}$ belong to the same component if and only if there is a chain of reflections $r=r_{1}, r_{2}, \ldots, r_{k}=r^{\prime}$ such that consecutive terms do not commute.

Lemma 14. Let $\alpha: W_{1} \rightarrow W_{2}$ be an isomorphism of finite rank Coxeter groups with $\alpha\left(\operatorname{Ref}\left(W_{1}\right)\right) \subseteq \operatorname{Ref}\left(W_{2}\right)$, and let $r, r^{\prime} \in \operatorname{Ref}\left(W_{1}\right)$. If $r$ and $r^{\prime}$ belong to the same component of $W_{1}$ then $\alpha(r)$ and $\alpha\left(r^{\prime}\right)$ belong to the same component of $W_{2}$.

Proof. This follows from the discussion above, since the image of a non-commuting chain from $r$ to $r^{\prime}$ is a non-commuting chain from $\alpha(r)$ to $\alpha\left(r^{\prime}\right)$.

Clearly symmetries of the Coxeter diagram give rise to automorphisms that permute the simple reflections; we call these graph automorphisms. We say that an automorphism is inner by graph if it lies in the subgroup of $\operatorname{Aut}(W)$ generated by the inner automorphisms and the graph automorphisms.

Note that since every reflection in $W$ is conjugate to a simple reflection, there are only finitely many conjugacy classes of reflections. Moreover, it is clear that if $\alpha$ is an automorphism and $C, C^{\prime}$ conjugacy classes such that $\alpha(C) \subseteq C^{\prime}$, then $\alpha(C)=C^{\prime}$. So if $\alpha$ preserves reflections, in the sense that $\alpha(\operatorname{Ref}(W)) \subseteq \operatorname{Ref}(W))$, then $\alpha(\operatorname{Ref}(W))=\operatorname{Ref}(W)$. In particular, $\alpha^{-1}$ also preserves reflections. 
We denote by $R(W)$ the set of all automorphisms of $W$ that preserve reflections. In view of the reasoning above we see that $R(W)$ is a subgroup of $\operatorname{Aut}(W)$. Clearly $R(W)$ includes all automorphisms that are inner by graph.

Given $\alpha \in R(W)$ there exists a function $\phi_{\alpha}: \Pi \rightarrow \Phi$ such that $\alpha$ maps the reflection along $a$ to the reflection along $\phi_{\alpha}(a)$, for all $a \in \Pi$. Note that $\phi_{\alpha}$ is not uniquely determined by $\alpha$; indeed, since $r_{b}=r_{c}$ if and only if $b= \pm c$ (given that $b, c \in \Phi$ ), there are exactly two choices for each $\phi_{\alpha}(a)$. Since the reflections $\left\{r_{b} \mid b \in \phi_{\alpha}(\Pi)\right\}$ generate $W$, the roots in $\phi_{\alpha}(\Pi)$ must span $V$ (by [10, Lemma 2.8]). Hence $\phi_{\alpha}(\Pi)$ is a basis of $V$.

If $a, b \in \Pi$ then $r_{a} r_{b}$ has order $m=m_{a b}$ and $a \cdot b=\cos (\pi / m)$. So if $\phi_{\alpha}(a)=c$ and $\phi_{\alpha}(b)=d$ then $r_{c} r_{d}=\alpha\left(r_{a} r_{b}\right)$ has order $m$. Since $r_{c} r_{d}$ acts as a rotation on the plane spanned by $c$ and $d$, we deduce that

$$
\phi_{\alpha}(a) \cdot \phi_{\alpha}(b)=\cos \left(l \pi / m_{a b}\right)
$$

for some $l$ coprime to $m_{a b}$. In particular, $\phi_{\alpha}(a) \cdot \phi_{\alpha}(b)=0$ if $m=2$.

Lemma 15. Let $\alpha \in R(W)$, and suppose that $\Gamma$, the Coxeter diagram of $W$, is a forest. Then the function $\phi_{\alpha}$ above can be chosen so that $\phi_{\alpha}(a) \cdot \phi_{\alpha}(b) \leq 0$ for all distinct $a, b \in \Pi$.

Proof. Observe that we can write $\Pi=\left\{a_{1}, a_{2}, \ldots, a_{n}\right\}$, choosing the numbering so that for each $i$ the valency of $a_{i}$ in the diagram associated with the subset $\left\{a_{1}, a_{2}, \ldots, a_{i}\right\}$ is at most 1 . If $b_{1}, b_{2}, \ldots, b_{n}$ are chosen arbitrarily subject to $\alpha\left(r_{a_{i}}\right)=r_{b_{i}}$, then for each $i$ there is at most one $j<i$ such that $b_{i} \cdot b_{j} \neq 0$, and we can successively choose signs $\varepsilon_{1}, \varepsilon_{2}, \ldots, \varepsilon_{n}$ so that $\left(\varepsilon_{i} b_{i}\right) \cdot\left(\varepsilon_{j} b_{j}\right) \leq 0$ whenever $i \neq j$.

It is not necessarily true that $\phi_{\alpha}(a) \cdot \phi_{\alpha}(b)=a \cdot b$, even if they agree in sign. However, if $m=2,3,4$ or 6 , then the only numbers $l \in\{1,2, \ldots, m-1\}$ coprime to $m$ are $l=1$ and $l=m-1$, and $\cos ((m-1) \pi / m)$ and $\cos (\pi / m)$ have opposite signs. Hence we deduce the following result.

Corollary 16. Suppose that $\alpha \in R(W)$ and $\Gamma$ is a forest with edge labels in the set $\{3,4,6\}$. Then we can choose $\phi_{\alpha}$ so that $\phi_{\alpha}(a) \cdot \phi_{\alpha}(b)=a \cdot b$ for all $a, b \in \Pi$.

The next result is an unpublished theorem of J-Y Hée. It follows immediately from [10, Theorem 4.1].

Theorem 17. Suppose that $W_{1}, W_{2}$ are irreducible Coxeter groups, with root systems $\Phi_{1}, \Phi_{2}$ and sets of simple roots $\Pi_{1}, \Pi_{2}$ in the spaces $V_{1}, V_{2}$. Suppose that $g: V_{1} \rightarrow V_{2}$ is linear, maps $\Phi_{1}$ to $\Phi_{2}$ bijectively, and satisfies $(g u) \cdot(g v)=u \cdot v$ for all $u, v \in V_{1}$. Then there exists $w \in W_{2}$ and $\varepsilon= \pm 1$ such that $g \Pi_{1}=\varepsilon w \Pi_{2}$.

Clearly $g \Pi_{1}=\varepsilon w \Pi_{2}$ implies that the Coxeter diagrams of $W_{1}$ and $W_{2}$ are isomorphic. In the case $W_{1}=W_{2}$ we see that the automorphism $x \mapsto g^{-1} x g$ is inner by graph.

Theorem 18. Suppose that $\alpha \in R(W)$ and suppose that the function $\phi_{\alpha}$ can be chosen so that $\phi_{\alpha}(a) \cdot \phi_{\alpha}(b)=a \cdot b$ for all $a, b \in \Pi$. Then $\alpha$ is inner by graph.

Proof. The function $\phi_{\alpha}: \Pi \rightarrow \Phi$ extends uniquely to a linear map $g: V \rightarrow V$. The hypothesis that $\phi_{\alpha}(a) \cdot \phi_{\alpha}(b)=a \cdot b$ for all $a, b \in \Pi$ ensures that $(g u) \cdot(g v)=u \cdot v$ for all $u, v \in V$.

Let $W_{1}, W_{2}, \ldots, W_{m}$ be the irreducible components of $W$, and $L_{1}, L_{2}, \ldots, L_{m}$ the corresponding subsets of $\Pi$. Write $V_{j}$ for the subspace of $V$ spanned by $L_{j}$. By Lemma 14 the sets $\operatorname{Ref}\left(W_{1}\right), \operatorname{Ref}\left(W_{2}\right), \ldots, \operatorname{Ref}\left(W_{m}\right)$ are permuted by $\alpha$. Now if $i, j \in\{1,2, \ldots, m\}$ satisfy $\alpha\left(\operatorname{Ref}\left(W_{i}\right)=\operatorname{Ref}\left(W_{j}\right)\right.$ then $\phi_{\alpha}(a) \in V_{j}$ for all $a \in L_{i}$, and so $g$ restricts to a linear map $V_{i} \rightarrow V_{j}$. Moreover, $g$ maps the root system of $W_{i}$ bijectively onto the root system of $W_{j}$, since $\alpha$ is bijective. Hence Theorem 17 applies, and we conclude that there exists $w_{j} \in W_{j}$ and $\varepsilon_{j}= \pm 1$ such that $\varepsilon_{j} w_{j}^{-1} \phi_{\alpha}(a) \in L_{j}$ for all $a \in L_{i}$. Repeating this construction 
for all values of $j$ yields a bijective map $\theta: \Pi \rightarrow \Pi$ such that $\varepsilon_{j} w_{j}^{-1} \theta(a)=\phi_{\alpha}(a)$ when $\theta(a) \in L_{j}$.

If $a, b \in \Pi$ belong to different components then so do $\theta(a)$ and $\theta(b)$, while if they belong to the same $L_{i}$ then

$$
\theta(a) \cdot \theta(b)=w_{j} \theta(a) \cdot w_{j} \theta(b)=\varepsilon_{j} w_{j} \theta(a) \cdot \varepsilon_{j} w_{j} \theta(b)=\phi_{\alpha}(a) \cdot \phi_{\alpha}(b)=a \cdot b .
$$

So in all cases we must have that $m_{\theta(a) \theta(b)}=m_{a b}$, whence $\theta$ gives rise to a graph automorphism of $W$. We denote this graph automorphism by $\gamma$.

Let $a \in \Pi$, and define $b \in \Phi$ and $j \in\{1,2, \ldots, m\}$ by $b=\phi_{\alpha}(a)$ and $\theta(a) \in L_{j}$. Then $b=\varepsilon_{j} w_{j}^{-1} \theta(a) \in \operatorname{Ref}\left(W_{j}\right)$, and we have

$$
\gamma\left(r_{a}\right)=r_{\theta(a)}=r_{\varepsilon_{j} w_{j} b}=r_{w_{j} b}=w_{j} r_{b} w_{j}^{-1}=\left(w_{1} w_{2} \cdots w_{m}\right) r_{b}\left(w_{1} w_{2} \cdots w_{m}\right)^{-1}
$$

since $w_{1}, w_{2}, \ldots, w_{m}$ centralize each other, and $w_{i}$ centralizes $r_{b}$ when $i \neq j$. But $r_{b}=\alpha\left(r_{a}\right)$ (since $b=\phi_{\alpha}(a)$ ), and so, writing $w=w_{1} w_{2} \cdots w_{m}$, we deduce that $\gamma\left(r_{a}\right)=w \alpha\left(r_{a}\right) w^{-1}$ for all $a \in \Pi$. Since the $r_{a}$ generate $W$ it follows that $\alpha(x)=w^{-1} \gamma(x) w$ for all $x \in W$, whence $\alpha$ is inner by graph.

Corollary 19. If the Coxeter diagram is a forest whose edge labels all belong to the set $\{3,4,6\}$, then all automorphisms of $W$ that preserve reflections are inner by graph.

Proof. This follows immediately from Theorem 18 and Corollary 16.

\section{NEARly Finite COXETER GROUPS}

Recall our definition of "nearly finite": a Coxeter group of rank $n$ is nearly finite if it is infinite and has a finite parabolic subgroup of rank $n-1$. In this section we begin our investigation of nearly finite Coxeter groups and their automorphisms. We show, in particular, that if $W$ is irreducible and nearly finite, and $\alpha$ is an automorphism of $W$ whose restriction to a finite subgroup of rank $n-1$ is inner by graph, then $\alpha$ itself is inner by graph.

If $W$ is a Coxeter group and $\Pi$ its set of simple roots, then we shall say that a subset $J$ of $\Pi$ is of finite type if the corresponding standard parabolic subgroup $W_{J}$ is finite.

The $n \times n$ symmetric matrix $M$ is reducible if there are non-empty sets $I$ and $J$ such that $I \cup J=\{1, \ldots, n\}$ and the $(i, j)$-entry of $M$ is zero for all $i \in I$ and $j \in J$. Otherwise $M$ is irreducible. We define the Gram matrix of the Coxeter group $W$ to be the $n \times n$ matrix whose $(i, j)$-entry is $a_{i} \cdot a_{j}$, where $\Pi=\left\{a_{1}, a_{2}, \ldots, a_{n}\right\}$. Clearly, the Gram matrix of $W$ is irreducible if and only if $W$ is irreducible. We say that $W$ is nondegenerate if the Gram matrix is nonsingular. Note that if $W$ is finite then it is nondegenerate, since the Gram matrix is positive definite (by Lemma 1 ).

Lemma 20. Suppose that $M$ is a positive definite real symmetric matrix such that the off-diagonal entries of $M$ are nonpositive, and let $Q=M^{-1}$. Then all entries of $Q$ are nonnegative. Moreover, if $M$ is irreducible then all entries of $Q$ are strictly positive.

Proof. Let $n$ be the degree of $M$, and write $m_{i j}$ and $q_{i j}$ for the $(i, j)$-entries of $M$ and $Q$, for all $i, j \in\{1,2, \ldots, n\}$. Let $e_{i}$ be the $i$-th vector in the standard basis of $\mathbb{R}^{n}$, written as a column vector, and let $v_{i}$ be the $i$-th column of $Q$. Note that since $M$ is symmetric, so too is $Q$. Hence $v_{i}^{\mathrm{t}}$ is the $i$-th row of $Q$ (where the " $\mathrm{t}$ " means "transpose").

The principal minors of $M$ are all positive, since $M$ is positive definite, and $q_{i i}$ equals the $(i, i)$-th cofactor of $M$ divided by the determinant of $M$. So it follows that $q_{i i}>0$ for all $i$.

Fix $k \in\{1,2, \ldots, n\}$, and define

$$
\begin{aligned}
& I=\left\{i \mid 1 \leq i \leq n \text { and } q_{i k} \geq 0\right\}, \\
& J=\left\{i \mid 1 \leq i \leq n \text { and } q_{i k}<0\right\} .
\end{aligned}
$$


Let $x=\sum_{i \in I} q_{i k} e_{i}$ and $y=\sum_{i \in J} q_{i k} e_{i}$, and observe that $x+y=v_{k}$. Now $v_{k}^{\mathrm{t}} M=e_{k}^{\mathrm{t}}$, since $Q=M^{-1}$, and so $v_{k}^{\mathrm{t}} M y$ is the $k$-th entry of $y$. But all the entries of $y$ are nonpositive; so

$$
0 \geq v_{k}^{\mathrm{t}} M y=y^{\mathrm{t}} M y+x^{\mathrm{t}} M y=y^{\mathrm{t}} M y+\sum_{\substack{i \in I \\ j \in J}} q_{i k} q_{j k} e_{i}^{\mathrm{t}} M e_{j}=y^{\mathrm{t}} M y+\sum_{\substack{i \in I \\ j \in J}} q_{i k} q_{j k} m_{i j} .
$$

Each term in this last sum is nonnegative, since $i \in I$ gives $q_{i k} \geq 0$ and $j \in J$ gives $q_{j k}<0$, while $m_{i j} \leq 0$ since $i \neq j$. Hence

$$
0 \geq y^{\mathrm{t}} M y+\sum_{I, J} q_{i k} q_{j k} m_{i j} \geq y^{\mathrm{t}} M y,
$$

and since $M$ is positive definite it follows that $y=0$. Hence $v_{k}=x$, and so all entries of $v_{k}$ are nonnegative. This applies for all $k$; so the entries of $Q$ are all nonnegative.

Suppose that $Q$ has at least one zero entry; say $q_{h k}=0$. Let $I=\left\{i \mid q_{h i}>0\right\}$ and $J=\left\{j \mid q_{h j}=0\right\}$. Then $I \cup J=\{1,2, \ldots, n\}$, since $q_{i j} \geq 0$ for all $i$ and $j$. Our hypothesis says that $k \in J$, whereas $h \in I$, since we proved above that $q_{h h}>0$. Hence both $I$ and $J$ are nonempty. Furthermore, if $j \in J$ then $j \neq h$, and we have

$$
0=e_{h}^{\mathrm{t}} e_{j}=v_{h}^{\mathrm{t}} M e_{j}=\sum_{i \in I} q_{h i} e_{i}^{\mathrm{t}} M e_{j}=\sum_{i \in I} q_{h i} m_{i j} .
$$

Note that $m_{i j}<0$ for all $i \in I$, since $j \notin I$, and since $q_{h i}>0$ for all $i \in I$ we see that all the terms $q_{h i} m_{i j}$ in the above sum are nonpositive. So they must all be zero. Since $q_{h i}>0$ for all $i \in I$, we deduce that $m_{i j}=0$ for all $i \in I$. Thus $m_{i j}=0$ for all $i \in I$ and $j \in J$, and hence $M$ is reducible.

Corollary 21. Suppose that $W$ is a finite Coxeter group, and let $\left(\xi_{a}\right)_{a \in \Pi}$ be a family of nonnegative real numbers indexed by the set $\Pi$ of simple roots. Then there exist nonnegative numbers $\left(\mu_{a}\right)_{a \in \Pi}$ such that $x=\sum_{a \in \Pi} \mu_{a}$ a satisfies $x \cdot a=\xi_{a}$ for all $a \in \Pi$. If $\xi_{a}>0$ for some $a \in \Pi$ then $\mu_{b}>0$ for all $b$ in the same component of $\Pi$ as $a$.

In particular, if $W$ is irreducible and $\xi_{a}>0$ for some $a$, then $\mu_{b}>0$ for all $b$.

Proof. Let $L$ be an irreducible component of $\Pi$, and let $M$ be the Gram matrix of $W_{L}$. Then $M$ is positive definite, by Lemma 1 , and the off-diagonal entries of $M$ are non-positive since $a \cdot b=-\cos \left(\pi / m_{a b}\right) \leq 0$ whenever $a, b \in \Pi$ with $a \neq b$. Furthermore, $M$ is irreducible since $W_{L}$ is irreducible. By Lemma 20 the entries of $M^{-1}$ are all positive.

Writing $q_{b c}$ for the $(b, c)$-entry of $M^{-1}$, define $\mu_{c}=\sum_{b \in L} q_{b c} \xi_{b}$ for each $c \in L$. Then $\mu_{c} \geq 0$, and $\mu_{c}>0$ if any $\xi_{b}$ is nonzero. Furthermore, if $x_{L}=\sum_{c \in L} \mu_{c} c$, then $x_{L} \cdot a=\xi_{a}$ for all $a \in L$. Repeating this construction for all components $L$, and defining $x=\sum_{L} x_{L}$, we see that $x \cdot a=\xi_{a}$ for all $a \in \Pi$, since distinct components are orthogonal to each other; moreover, $x=\sum_{a \in \Pi} \mu_{a} a$ with coefficients $\mu_{a}$ that are nonnegative, and positive when $\xi_{c} \neq 0$ for some $c$ in the same component as $a$.

We shall make use of the following triviality.

Lemma 22. Suppose that $W$ is a Coxeter group, $\alpha$ an automorphism of $W$, and $a, b, c$ and $d$ simple roots such that $\alpha\left(r_{a}\right)=r_{c}$ and $\alpha\left(r_{b}\right)=r_{d}$. Then $a \cdot b=c \cdot d$.

Proof. We have $a \cdot b=-\cos \left(\pi / m_{a b}\right)$ and $c \cdot d=-\cos \left(\pi / m_{c d}\right)$, where $m_{a b}$ and $m_{c d}$ are the orders of $r_{a} r_{b}$ and $r_{c} r_{d}$. But these orders are equal since $\alpha\left(r_{a} r_{b}\right)=r_{c} r_{d}$.

We now come to the main result of this section.

Theorem 23. Suppose that $W$ is irreducible, non-degenerate and nearly finite, and the Coxeter diagram of has no infinite edge labels. Let $S$ be the set of simple reflections. Suppose that $\alpha$ is an automorphism of $W$ that preserves reflections, and suppose that there exist $a, b \in \Pi$ (possibly equal) such that $\Pi \backslash\{a\}$ and $\Pi \backslash\{b\}$ are of finite type and $\alpha\left(S \backslash\left\{r_{a}\right\}\right)=S \backslash\left\{r_{b}\right\}$. Then $\alpha$ is inner by graph. 
Proof. Let $V_{a}$ and $V_{b}$ be the subspaces of $V$ spanned by $\Pi \backslash\{a\}$ and $\Pi \backslash\{b\}$. Let $x \in V_{a}$ be such that the vector $u=x+a$ lies in the orthogonal complement of $V_{a}$ (which exists since $W$ is nondegenerate), and, similarly, let $y \in V_{b}$ be such that the vector $v=y+b$ lies in the orthogonal complement of $V_{b}$. We shall show that $y \cdot y \leq x \cdot x$. Since the same argument with $a$ and $b$ interchanged and $\alpha$ replaced by $\alpha^{-1}$ will show that $x \cdot x \leq y \cdot y$, it will follow that $x \cdot x=y \cdot y$.

Write $\Pi \backslash\{a\}=J_{1} \cup J_{2} \cup \cdots \cup J_{k}$, where the $J_{j}$ are the irreducible components of $\Pi \backslash\{a\}$. For each $c \in \Pi \backslash\{a\}$ define

$$
\xi_{c}=\cos \left(\pi / m_{c a}\right)=-c \cdot a,
$$

and observe that, since $W$ is irreducible, each $J_{j}$ contains at least one $c$ such that $\xi_{c} \neq 0$. Now $x$ is the orthogonal projection of $-a$ onto $V_{a}$, and since the sets $J_{j}$ are mutually orthogonal it follows that

$$
x=x_{1}+x_{2}+\cdots+x_{k}
$$

where $x_{j}$ is the orthogonal projection of $-a$ onto the subspace spanned by $J_{j}$. For all $c \in J_{j}$ we have

$$
c \cdot x_{j}=-c \cdot a=\xi_{c} \geq 0,
$$

with strict inequality for at least one $c \in J_{j}$, and so if we write $x_{j}=\sum_{c \in J_{j}} \mu_{c} c$ then it follows from Corollary 21 that $\mu_{c}>0$ for all $c \in J_{j}$. Thus $x=\sum_{c \in \Pi \backslash\{a\}} \mu_{c} c$ with all coefficients $\mu_{c}$ positive.

Since $\alpha\left(S \backslash\left\{r_{a}\right\}\right)=S \backslash\left\{r_{b}\right\}$ there is a bijection $\sigma: \Pi \backslash\{a\} \rightarrow \Pi \backslash\{b\}$ with $\alpha\left(r_{c}\right)=r_{\sigma(c)}$ for all $c \in \Pi \backslash\{a\}$. By Lemma 22 we have $c \cdot d=\sigma(c) \cdot \sigma(d)$ for all $c, d \in \Pi \backslash\{a\}$, and extending $\sigma$ linearly gives an isomorphism $\widetilde{\sigma}: V_{a} \rightarrow V_{b}$.

Let $f \in \Phi^{+}$be such that $\alpha\left(r_{a}\right)=r_{f}$. Since $r_{\sigma(c)} r_{f}$ has the same order as $r_{c} r_{a}$, namely $m_{c a}$, we have that

$$
f \cdot \sigma(c)=\cos \left(l_{c} \pi / m_{c a}\right)
$$

for some $l_{c}$ coprime to $m_{c a}$. Write $\theta_{c}=\cos \left(l_{c} \pi / m_{c a}\right)$, and note that $\xi_{c} \geq\left|\theta_{c}\right|$ for all $c \in \Pi \backslash\{a\}$, with $\theta_{c}=0$ if and only if $\xi_{c}=0$.

Let $z=\sum_{c \in \Pi \backslash\{b\}} \lambda_{c} c$ be the orthogonal projection of $f$ onto $V_{b}$, so that

$$
\sigma(c) \cdot z=\sigma(c) \cdot f=\theta_{c}
$$

for all $c \in \Pi \backslash\{a\}$. Since $v=b+y$ is a nonzero element of the orthogonal complement of $V_{b}$ we have that $f=z+\omega v$ for some scalar $\omega$. Now since $f=\omega b+(z+\omega y)$ and $z+\omega y \in V_{b}$, it follows from Lemma 3 that $\omega \geq 1$. Note also that

$$
z=z_{1}+z_{2}+\cdots+z_{k}
$$

where $z_{j}=\sum_{c \in J_{j}} \lambda_{\sigma(c)} \sigma(c)$ is the projection of $f$ onto the space spanned by $\sigma\left(J_{j}\right)$.

Fix an arbitrary $j \in\{1,2, \ldots, k\}$. Since $\xi_{c} \geq\left|\theta_{c}\right|$ for all $c \in J_{j}$, we have

$$
\begin{aligned}
0 \leq \xi_{c}-\theta_{c}=c \cdot x-\sigma(c) \cdot z & =c \cdot\left(\sum_{d \in J_{j}} \mu_{d} d\right)-\sigma(c) \cdot\left(\sum_{d \in J_{j}} \lambda_{\sigma(d)} \sigma(d)\right) \\
& =\sum_{d \in J_{j}}(c \cdot d) \mu_{d}-\sum_{d \in J_{j}}(c \cdot d) \lambda_{\sigma(d)}=\sum_{d \in J_{j}}(c \cdot d)\left(\mu_{d}-\lambda_{\sigma(d)}\right),
\end{aligned}
$$

since $\sigma(c) \cdot \sigma(d)=c \cdot d$ for all $c, d \in J_{j}$. Now by Corollary 21 it follows that $\mu_{d}-\lambda_{\sigma(d)} \geq 0$ for all $d \in J_{j}$, and, moreover, if $\mu_{d}-\lambda_{\sigma(d)}=0$ for some $d \in J_{j}$ then we must have $\xi_{c}-\theta_{c}=0$ for all $c \in J_{j}$. Similarly,

$$
0 \leq \xi_{c}+\theta_{c}=\sum_{d \in J_{j}}(c \cdot d)\left(\mu_{d}+\lambda_{\sigma(d)}\right)
$$


for all $c \in J_{j}$; so $\mu_{d}+\lambda_{\sigma(d)} \geq 0$ for all $d \in J_{j}$, equality occurring for some $d$ only if $\xi_{c}+\theta_{c}=0$ for all $c \in J_{j}$. Note in particular that, since $j$ is arbitrary in the above argument, $\mu_{d} \geq\left|\lambda_{\sigma(d)}\right|$ for all $d \in \Pi \backslash\{a\}$.

Each $t \in V$ can be written in the form $t=t_{0}+v u$ with $t_{0} \in V_{a}$ and $v \in \mathbb{R}$. If $u \cdot u>0$ this gives

$$
t \cdot t=t_{0} \cdot t_{0}+v^{2} u \cdot u
$$

which is positive if $t_{0} \neq 0$ or if $v \neq 0$. Since $W$ is infinite this contradicts Part (3) of Lemma 1. If $u \cdot u=0$ then $t \cdot u=0$ for all $t \in V$, contrary to the assumption that $W$ is nondegenerate. So $u \cdot u<0$, and, by the same reasoning, $v \cdot v<0$.

Since $f \in \Phi$

$$
1=f \cdot f=(z+\omega v) \cdot(z+\omega v)=z \cdot z+\omega^{2} v \cdot v,
$$

and we also have that

$$
z \cdot z=\sum_{c \in \Pi \backslash\{a\}} \lambda_{\sigma(c)} \sigma(c) \cdot z=\sum_{c \in \Pi \backslash\{a\}} \lambda_{\sigma(c)} \theta_{c} .
$$

Similarly,

$$
1=a \cdot a=(-x+u) \cdot(-x+u)=x \cdot x+u \cdot u,
$$

and also

$$
x \cdot x=\sum_{c \in \Pi \backslash\{a\}} \mu_{c} c \cdot x=\sum_{c \in \Pi \backslash\{a\}} \mu_{c} \xi_{c} .
$$

Thus

$$
u \cdot u+\sum_{c \in \Pi \backslash\{a\}} \mu_{c} \xi_{c}=\omega^{2} v \cdot v+\sum_{c \in \Pi \backslash\{a\}} \lambda_{\sigma(c)} \theta_{c},
$$

and so

$$
\sum_{c \in \Pi \backslash\{a\}}\left(\mu_{c} \xi_{c}-\lambda_{\sigma(c)} \theta_{c}\right)=\omega^{2} v \cdot v-u \cdot u .
$$

Since $\mu_{c} \geq\left|\lambda_{\sigma(c)}\right|$ and $\xi_{c} \geq\left|\theta_{c}\right|$ for all $c$, we see that $\sum_{c \in \Pi \backslash\{a\}}\left(\mu_{c} \xi_{c}-\lambda_{\sigma(c)} \theta_{c}\right) \geq 0$, and so $\omega^{2} v \cdot v \geq u \cdot u$. But $\omega^{2} \geq 1$, and since $v \cdot v<0$ it follows that $v \cdot v \geq \omega^{2} v \cdot v$, and hence $v \cdot v \geq u \cdot u$. Since $1=x \cdot x+u \cdot u$ (shown above) and $1=y \cdot y+v \cdot v$ (similarly), it follows that $y \cdot y \leq x \cdot x$, as desired.

In view of our earlier remarks, we must have $v \cdot v=u \cdot u$, and

$$
0 \leq \sum_{c \in \Pi \backslash\{a\}}\left(\mu_{c} \xi_{c}-\lambda_{\sigma(c)} \theta_{c}\right)=\left(\omega^{2}-1\right) u \cdot u \leq 0
$$

since $\omega \geq 1$ and $u \cdot u<0$. Thus $\left(\omega^{2}-1\right) u \cdot u=0$, giving $\omega=1$, and

$$
\sum_{c \in \Pi \backslash\{a\}}\left(\mu_{c} \xi_{c}-\lambda_{\sigma(c)} \theta_{c}\right)=0,
$$

giving $\mu_{c} \xi_{c}=\lambda_{\sigma(c)} \theta_{c}=\left|\lambda_{\sigma(c)} \theta_{c}\right|$ for all $c \in \Pi \backslash\{a\}$. Furthermore, we have

$$
0 \leq\left(\mu_{c}-\left|\lambda_{\sigma(c)}\right|\right) \xi_{c} \leq \mu_{c} \xi_{c}-\left|\lambda_{\sigma(c)}\right|\left|\theta_{c}\right|=0,
$$

and it follows that, for all $c \in \Pi \backslash\{a\}$, either $\xi_{c}=0$ or $\left|\lambda_{\sigma(c)}\right|=\mu_{c}$. As noted above, for each $j \in\{1,2, \ldots, k\}$ there exists at least one $d \in J_{j}$ with $\xi_{d}>0$, so that $\lambda_{\sigma(d)}= \pm \mu_{d}$. But as we have shown, if $\lambda_{\sigma(d)}=\mu_{d}$ then $\theta_{c}=\xi_{c}$ for all $c \in J_{j}$, and if $\lambda_{\sigma(d)}=-\mu_{d}$ then $\theta_{c}=-\xi_{c}$ for all $c \in J_{j}$. In the former case we have

$$
z_{j} \cdot \sigma(c)=\theta_{c}=\xi_{c}=x_{j} \cdot c
$$

for all $c \in J_{j}$, and it follows that $z_{j}=\tilde{\sigma}\left(x_{j}\right)$. In the latter case,

for all $c \in J_{j}$, giving $z_{j}=-\widetilde{\sigma}\left(x_{j}\right)$.

$$
z_{j} \cdot \sigma(c)=\theta_{c}=-\xi_{c}=-x_{j} \cdot c
$$

Let $w$ be the the longest element of the parabolic subgroup corresponding to the union of the sets $\sigma\left(J_{j}\right)$ for which $z_{j}=\widetilde{\sigma}\left(x_{j}\right)$, let $\beta$ be the inner automorphism of $W$ given by 
conjugation by $w$, and let $\alpha^{\prime}=\beta \alpha$. Since $\beta$ permutes $S \backslash\left\{r_{b}\right\}$ we see that $\alpha^{\prime}$ satisfies the same hypotheses as $\alpha$. Now $\alpha^{\prime}\left(r_{a}\right)=w r_{f} w^{-1}=r_{w f}$, and

$$
w f=w z+v=\left(w z_{1}+w z_{2}+\cdots+w z_{k}\right)+v,
$$

and here $w z_{j}$ is the projection of $w f$ onto the span of $\sigma\left(J_{j}\right)$. Applying to $\alpha^{\prime}$ the arguments used above for $\alpha$ enables us to deduce that for each $j$

$$
w z_{j}= \pm \tilde{\sigma}^{\prime}\left(x_{j}\right)= \pm \sum_{c \in J_{j}} \mu_{c} \sigma^{\prime}(c),
$$

where $\alpha^{\prime}\left(r_{c}\right)=r_{\sigma^{\prime}(c)}$ for all $c \in \Pi \backslash\{a\}$. But $w$ was chosen so that for each $j$ the element $w z_{j}$ is a negative linear combination simple roots, and so $w z_{j}=-\tilde{\sigma}^{\prime}\left(x_{j}\right)$. Thus

$$
w f=\left(-\tilde{\sigma}^{\prime}\left(x_{1}\right)-\tilde{\sigma}^{\prime}\left(x_{2}\right)-\cdots-\tilde{\sigma}^{\prime}\left(x_{k}\right)\right)+v=-\tilde{\sigma}^{\prime}(x)+v,
$$

showing that $w f \cdot \sigma^{\prime}(c)=-\xi_{c}=a \cdot c$ for all $c \in \Pi \backslash\{a\}$. Since also $\sigma^{\prime}(d) \cdot \sigma^{\prime}(c)=d \cdot c$ for all $c, d \in \Pi \backslash\{a\}$, Theorem 18 shows that $\alpha^{\prime}$ is inner by graph. (Indeed, Theorem 17 yields that there exists a $w^{\prime} \in W$ and $\varepsilon= \pm 1$ such that $\varepsilon w^{\prime}(\Pi)=w f \cup \Pi \backslash\{b\}$. But $w f$ is easily shown to be positive, and it follows readily that $\varepsilon=1$ and $N\left(w^{\prime}\right)=\emptyset$. Hence $w f=b$, and $\alpha^{\prime}$ is in fact a graph automorphism.)

Our main objective is to prove that Theorem 23 holds without the hypothesis that $\alpha\left(S \backslash\left\{r_{a}\right\}\right)=S \backslash\left\{r_{b}\right\}$. Our basic strategy is to show that if the given automorphism $\alpha$ is replaced by $\alpha \beta$ for some suitably chosen $\beta$ that is inner by graph, then the hypotheses of Theorem 23 are satisfied. Our next theorem accomplishes this in the case that $\alpha$ preserves some maximal finite subgroup. We need to use a modification of the argument used in the proof of Theorem 23 to deal with some of the cases.

Theorem 24. Suppose that $W$ is irreducible, non-degenerate and nearly finite, and the Coxeter diagram has no infinite edge labels. Suppose that $\alpha$ is an automorphism of $W$ that preserves reflections, and suppose that there exists $a \in \Pi$ such that $\alpha\left(W_{\Pi \backslash\{a\}}\right)=W_{\Pi \backslash\{a\}}$. Then $\alpha$ is inner by graph.

Proof. Write $J=\Pi \backslash\{a\}$. Since $W_{J}$ is finite, the classification of finite Coxeter groups (see [11, Section 2.7]) tells us that each irreducible component of $J$ is of one of the types in the following list. As is customary, labels equal to 3 are suppressed.
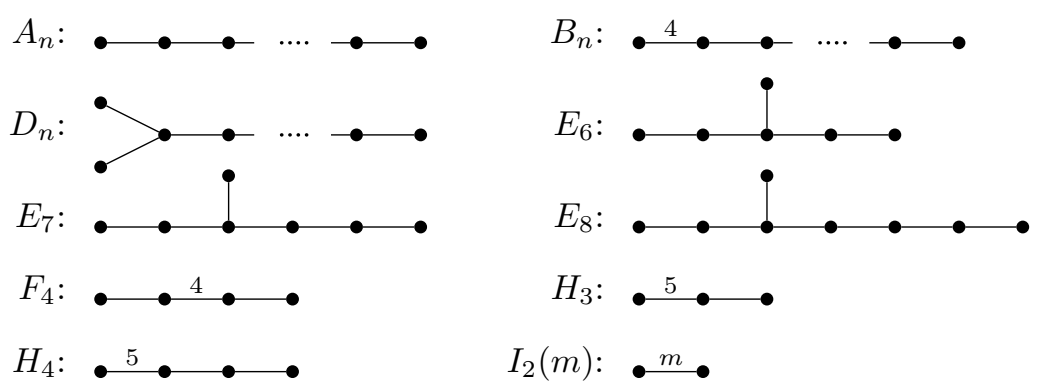

Thus the Coxeter diagram of $W_{J}$ is a forest, and so by Lemma 15 there is a function $\phi_{\alpha}: J \rightarrow \Phi$ such that $\alpha\left(r_{b}\right)=r_{\phi_{\alpha}(b)}$ for all $b \in J$ and $\phi_{\alpha}(b) \cdot \phi_{\alpha}(c) \leq 0$ for all $b, c \in J$ with $b \neq c$. As in Corollary 16 it follows that

$$
\phi_{\alpha}(b) \cdot \phi_{\alpha}(c)=b \cdot c
$$

unless $m_{b c}=5$ or $m_{b c} \geq 7$. Furthermore, these values for $m_{b c}$ can only occur if $b$ and $c$ lie in an irreducible component of $J$ of type $H_{3}, H_{4}$ or $I_{2}(m)$, and then only for one pair of simple roots in the component.

If Eq. (3.1) does hold for all $b, c \in J$ then by Theorem 17 there exists $w \in W_{J}$ and $\varepsilon= \pm 1$ such that $\varepsilon w \phi_{\alpha}(b) \in J$ for all $b \in J$, and if $\beta$ is the inner automorphism of $W$ given 
by $x \mapsto w x w^{-1}$ then we see that $\beta \alpha$ permutes the simple reflections of $W_{J}$. Theorem 23 can then be applied, and it follows that $\beta \alpha$ is inner by graph, whence $\alpha$ is also inner by graph.

It remains to deal with those cases in which $J$ has at least one irreducible component of type $H_{3}, H_{4}$ or $I_{2}(m)$ (where $m=5$ or $m \geq 7$ ) on which Eq. 3.1) does not hold. Accordingly, assume that $J$ has such a component. We use an argument similar to that used in the proof of Theorem 23 to derive a contradiction.

In our calculations below we use the abbreviations $\mathrm{c}(\theta)$ and $\mathrm{s}(\theta)$ for $\cos (\theta)$ and $\sin (\theta)$, and we also write $\pi_{m}$ for $\pi / m$.

If $b, c \in J$ are such that Eq. (3.1) fails, then $b \cdot c=-\mathrm{c}\left(\pi_{m}\right)$, where $m=m_{b c}$, and $\phi_{\alpha}(b) \cdot \phi_{\alpha}(c)=-\mathrm{c}\left(j \pi_{m}\right)$ for some $j$ coprime to $m$. Since $\phi_{\alpha}(b) \cdot \phi_{\alpha}(c) \leq 0$ we have that $1<j<m / 2$. If the component of $J$ containing $b$ and $c$ is of type $H_{3}$ or $H_{4}$ then $m=5$ and $j=2$.

Let $M$ and $M^{\prime}$ be matrices with rows and columns indexed by $J$, such that, for all $b, c \in J$, the $(b, c)$-entry of $M$ is $b \cdot c$ and the $(b, c)$-entry of $M^{\prime}$ is $\phi_{\alpha}(b) \cdot \phi_{\alpha}(c)$. We assume that $J$ is ordered so that $M$ is a diagonal sum of matrices corresponding to the various irreducible components of $J$. Since $\phi_{\alpha}(b) \cdot \phi_{\alpha}(c)=0$ if and only if $b \cdot c=0$, we see that $M^{\prime}$ is also a diagonal sum, with blocks of the same sizes as those of $M$.

The blocks of $M$ corresponding to components of types $I_{2}(m), H_{3}$ and $H_{4}$ are as follows (assuming the ordering is chosen appropriately).

$$
\begin{aligned}
M_{I} & =\left[\begin{array}{cc}
1 & -\mathrm{c}\left(\pi_{m}\right) \\
-\mathrm{c}\left(\pi_{m}\right) & 1
\end{array}\right] \\
M_{3} & =\left[\begin{array}{ccc}
1 & -\mathrm{c}\left(\pi_{5}\right) & 0 \\
-\mathrm{c}\left(\pi_{5}\right) & 1 & -1 / 2 \\
0 & -1 / 2 & 1
\end{array}\right] \\
M_{4} & =\left[\begin{array}{cccc}
1 & -\mathrm{c}\left(\pi_{5}\right) & 0 & 0 \\
-\mathrm{c}\left(\pi_{5}\right) & 1 & -1 / 2 & 0 \\
0 & -1 / 2 & 1 & -1 / 2 \\
0 & 0 & -1 / 2 & 1
\end{array}\right]
\end{aligned}
$$

The following matrices $T_{3}, T_{4}$ and $T_{I}$ are the inverses of $M_{3}, M_{4}$ and $M_{I}$.

$$
\begin{aligned}
T_{I}= & {\left[\begin{array}{cc}
1 / \mathrm{s}^{2}\left(\pi_{m}\right) & \mathrm{c}\left(\pi_{m}\right) / \mathrm{s}^{2}\left(\pi_{m}\right) \\
\mathrm{c}\left(\pi_{m}\right) / \mathrm{s}^{2}\left(\pi_{m}\right) & 1 / \mathrm{s}^{2}\left(\pi_{m}\right)
\end{array}\right] } \\
T_{3}= & {\left[\begin{array}{ccc}
\frac{9+3 \sqrt{5}}{2} & 4+2 \sqrt{5} & 2+\sqrt{5} \\
4+2 \sqrt{5} & 6+2 \sqrt{5} & 3+\sqrt{5} \\
2+\sqrt{5} & 3+\sqrt{5} & \frac{5+\sqrt{5}}{2}
\end{array}\right] } \\
T_{4}= & {\left[\begin{array}{cccc}
28+12 \sqrt{5} & 33+15 \sqrt{5} & 22+10 \sqrt{5} & 11+5 \sqrt{5} \\
33+15 \sqrt{5} & 42+18 \sqrt{5} & 28+12 \sqrt{5} & 14+6 \sqrt{5} \\
22+10 \sqrt{5} & 28+12 \sqrt{5} & 20+8 \sqrt{5} & 10+4 \sqrt{5} \\
11+5 \sqrt{5} & 14+6 \sqrt{5} & 10+4 \sqrt{5} & 6+2 \sqrt{5}
\end{array}\right] }
\end{aligned}
$$

For components on which Eq 3.1 fails, the corresponding blocks of $M^{\prime}$ are as follows.

$$
\begin{aligned}
M_{I}^{\prime} & =\left[\begin{array}{ccc}
1 & -\mathrm{c}\left(j \pi_{m}\right) \\
-\mathrm{c}\left(j \pi_{m}\right) & 1
\end{array}\right] \\
M_{3}^{\prime} & =\left[\begin{array}{ccc}
1 & -\mathrm{c}\left(2 \pi_{5}\right) & 0 \\
-\mathrm{c}\left(2 \pi_{5}\right) & 1 & -1 / 2 \\
0 & -1 / 2 & 1
\end{array}\right] \\
M_{4}^{\prime} & =\left[\begin{array}{cccc}
1 & -\mathrm{c}\left(2 \pi_{5}\right) & 0 & 0 \\
-\mathrm{c}\left(2 \pi_{5}\right) & 1 & -1 / 2 & 0 \\
0 & -1 / 2 & 1 & -1 / 2 \\
0 & 0 & -1 / 2 & 1
\end{array}\right]
\end{aligned}
$$


The corresponding inverses are as follows.

$$
\begin{aligned}
& T_{I}^{\prime}= {\left[\begin{array}{cc}
1 / \mathrm{s}^{2}\left(j \pi_{m}\right) & \mathrm{c}\left(j \pi_{m}\right) / \mathrm{s}^{2}\left(j \pi_{m}\right) \\
\mathrm{c}\left(j \pi_{m}\right) / \mathrm{s}^{2}\left(j \pi_{m}\right) & 1 / \mathrm{s}^{2}\left(j \pi_{m}\right)
\end{array}\right] } \\
& T_{3}^{\prime}= {\left[\begin{array}{ccc}
\frac{9-3 \sqrt{5}}{2} & -4+2 \sqrt{5} & -2+\sqrt{5} \\
-4+2 \sqrt{5} & 6-2 \sqrt{5} & 3-\sqrt{5} \\
-2+\sqrt{5} & 3-\sqrt{5} & \frac{5-\sqrt{5}}{2}
\end{array}\right] } \\
& T_{4}^{\prime}=\left[\begin{array}{cccc}
28-12 \sqrt{5} & -33+15 \sqrt{5} & -22+10 \sqrt{5} & -11+5 \sqrt{5} \\
-33+15 \sqrt{5} & 42-18 \sqrt{5} & 28-12 \sqrt{5} & 14-6 \sqrt{5} \\
-22+10 \sqrt{5} & 28-12 \sqrt{5} & 20-8 \sqrt{5} & 10-4 \sqrt{5} \\
-11+5 \sqrt{5} & 14-6 \sqrt{5} & 10-4 \sqrt{5} & 6-2 \sqrt{5}
\end{array}\right]
\end{aligned}
$$

It can be checked that all the entries of $T_{I}^{\prime}, T_{3}^{\prime}$ and $T_{4}^{\prime}$ are positive and strictly less than the corresponding entries of $T_{I}, T_{3}$ and $T_{4}$. Hence if we write $t_{b c}$ and $t_{b c}^{\prime}$ for the $(b, c)$-entries of $M^{-1}$ and $\left(M^{\prime}\right)^{-1}$, then we have $t_{b c} \geq t_{b c}^{\prime}$ for all $b, c \in J$. Since there is a component for which Eq. 3.1 fails, there is a block in which $t_{b c}>t_{b c}^{\prime}$ for all $b$ and $c$.

As in the proof of Theorem 23 , we suppose that $\alpha\left(r_{a}\right)=r_{f}$, where $f \in \Phi^{+}$, and let $z$ be the projection of $f$ onto $V_{J}$. Let $x$ be the projection of $-a$ onto $V_{J}$. Then $u=x+a$ spans the orthogonal complement of $V_{J}$ in $V$, and $u \cdot u<0$ since $W$ is non-degenerate and infinite. Moreover, $f=z+\omega u$ for some scalar $\omega$, and by Lemma 3 we have $\omega \geq 1$.

For each $c \in J$ let $c \cdot a=-\mathrm{c}\left(\pi_{m_{c a}}\right)=-\xi_{c}$. Write $x=\sum_{c \in J} \mu_{c} c$. For all $c \in J$,

$$
c \cdot x=-c \cdot a=\xi_{c},
$$

so that $\xi_{c}=\sum_{d \in J}(c \cdot d) \mu_{d}$, and

$$
\mu_{c}=\sum_{d \in J} t_{c d} \xi_{d}
$$

Now for each $c \in J$ there is an integer $j_{c}$ such that

$$
\phi_{\alpha}(c) \cdot z=\phi_{\alpha}(c) \cdot f=\mathrm{c}\left(j c \pi_{m_{c a}}\right)=\theta_{c},
$$

where $\left|\theta_{c}\right| \leq \xi_{c}$ and $\theta_{c}=0$ if and only if $\xi_{c}=0$. Writing $z=\sum_{c \in J} \lambda_{c} \phi_{\alpha}(c)$, we have

$$
\lambda_{c}=\sum_{d \in J} t_{c d}^{\prime} \theta_{d}
$$

for all $c \in J$. Now observe that

$$
z \cdot z=\sum_{c \in J} \lambda_{c}\left(\phi_{\alpha}(c) \cdot z\right)=\sum_{c \in J} \lambda_{c} \theta_{c}=\sum_{c \in J} \sum_{d \in J} t_{c d}^{\prime} \theta_{c} \theta_{d}
$$

and

$$
x \cdot a=\sum_{c \in J} \mu_{c}(c \cdot a)=-\sum_{c \in J} \mu_{c} \xi_{c}=-\sum_{c \in J} \sum_{d \in J} t_{c d} \xi_{c} \xi_{d} .
$$

Since $\xi_{c} \xi_{d} \geq\left|\theta_{c} \theta_{d}\right| \geq \theta_{c} \theta_{d}$ and $t_{c d} \geq t_{c d}^{\prime} \geq 0$ for all $c, d \in J$,

$$
\sum_{c} \sum_{d} t_{c d} \xi_{c} \xi_{d} \geq \sum_{c} \sum_{d} t_{c d}^{\prime} \theta_{c} \theta_{d}
$$

But there is an irreducible component of $W_{J}$ for which $t_{c d}>t_{c d}^{\prime}$. As $W$ is irreducible there is an edge joining $a$ to this component, and hence there is a $c$ in this component for which $\xi_{c}>0$. Then $t_{c c} \xi_{c}^{2} \geq t_{c c} \theta_{k}^{2}>t_{c c}^{\prime} \theta_{c}^{2}$, and so

$$
-x \cdot a=\sum_{c} \sum_{d} t_{c d} \xi_{c} \xi_{d}>\sum_{c} \sum_{d} t_{c d}^{\prime} \theta_{c} \theta_{d}=z \cdot z .
$$

Therefore $1+x \cdot a<1-z \cdot z$. Now

$$
u \cdot u=(x+a) \cdot u=a \cdot u=a \cdot a+a \cdot x=1+a \cdot x .
$$

Thus $u \cdot u<1-z \cdot z$, and, since $u \cdot u<0$,

$$
1>\frac{1-z \cdot z}{u \cdot u} .
$$


Since $x \in \Phi$,

$$
1=x \cdot x=(z+\omega u) \cdot(z+\omega u)=z \cdot z+\omega^{2} u \cdot u .
$$

Hence

$$
\omega^{2}=\frac{1-z \cdot z}{u \cdot u}<1
$$

But $\omega \geq 1$, and so we have obtained the desired contradiction.

\section{GROUPS WITH TWO FINITE MAXIMAL PARABOLIC SUBGROUPS}

If $\alpha \in \operatorname{Aut}(W)$ and $F$ is a maximal finite subgroup of $W$, then clearly $\alpha(F)$ is also a maximal finite subgroup of $W$. Theorem 24 was concerned with the case $\alpha(F)=F$; in this section we dispense with this assumption.

Proposition 25. Suppose that $\alpha: W \rightarrow W^{\prime}$ is an isomorphism of finite Coxeter groups that maps reflections to reflections. Then $W$ and $W^{\prime}$ have the same type.

Proof. Since the irreducible components of $W$ and $W^{\prime}$ are generated by the reflections they contain, it follows from Lemma 14 that $\alpha$ maps the components of $W$ to the components of $W^{\prime}$. Hence it is sufficient to prove the result for irreducible $W$ and $W^{\prime}$.

If $W$ is of type $I_{2}(\mathrm{~m})$ then exactly half the elements of $W$ are reflections, and since $\alpha$ maps reflections to reflections it follows that half the elements of $W^{\prime}$ are reflections. Since $I_{2}(m)$ is the only type of Coxeter group with this property, it follows that $W^{\prime}$ is of the same type as $W$. Of course a similar argument applies whenever $W^{\prime}$ is of type $I_{2}(m)$; so we may assume that neither $W$ nor $W^{\prime}$ is of type $I_{2}(m)$.

The only coincidences of order for finite irreducible Coxeter groups, excluding groups of type $I_{2}(m)$, occurs for types $A_{4}$ and $H_{3}$, which both have order 120 . They are not isomorphic, since, for example, $A_{4}$ has trivial centre while $H_{3}$ does not. Since $W$ and $W^{\prime}$ have the same order and are isomorphic, we conclude that they are of the same type.

Proposition 26. Suppose that $W$ is an irreducible nearly finite Coxeter group, and let $a \in \Pi$ be such that $\Pi \backslash\{a\}$ is of finite type. Suppose that there exists $b \in \Pi$ with $b \neq a$ and $\Pi \backslash\{b\}$ of the same type as $\Pi \backslash\{a\}$. If $\Pi \backslash\{a\}$ and $\Pi \backslash\{b\}$ have at least one component of type $\mathrm{H}_{3}, \mathrm{H}_{4}$ or $\mathrm{I}_{2}(\mathrm{~m})$ for $m>4$, then the Coxeter diagram associated with $W$ either has $a$ symmetry of order two that interchanges $a$ and $b$, or is of type $X(q)$ for some $q \geq 2$, where these diagrams are as follows:
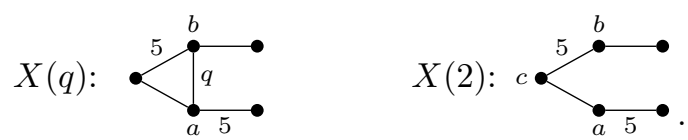

Proof. Let $\Gamma$ be the Coxeter diagram of $W$, and $\Gamma_{a}, \Gamma_{b}$ the diagrams obtained by deleting $a, b$ respectively. For each $c \in \Pi$ let $\operatorname{val}(c)$ be the valency of $c$ as a vertex of $\Gamma$. Observe that the valency of $c \neq a$ as a vertex of $\Gamma_{a}$ is $\operatorname{val}(c)-1$ or $\operatorname{val}(c)$ if $c$ is adjacent to $a$ or not adjacent to $a$ in $\Gamma$; so the sum of the valencies in $\Gamma_{a}$ is $\left(\sum_{c \in \Pi} \operatorname{val}(c)\right)-2 \operatorname{val}(a)$. Applying the same reasoning also to $\Gamma_{b}$ we deduce that $\operatorname{val}(a)=\operatorname{val}(b)$, since $\Gamma_{a}$ and $\Gamma_{b}$ are isomorphic.

Suppose first that $\Gamma_{a}$ and $\Gamma_{b}$ are reducible, and $b$ does not lie in a component of $\Gamma_{a}$ of type $H_{3}, H_{4}$ or $I_{2}(m)$ for $m>4$. Note that $\operatorname{val}(a) \geq 2$ since there must be edges from $a$ to all components of $\Gamma_{a}$.

By hypothesis $\Gamma_{a}$ has a component $\Delta$ of type $H_{3}, H_{4}$ or $I_{2}(m)$ that does not contain $b$. Observe that $\Delta$ lies in a component $\Delta^{\prime}$ of $\Gamma_{b}$ that also contains $a$, since $a$ is connected to $\Delta$. Since $H_{4}$ and $I_{2}(m)$ for $m>5$ are not contained in any larger diagrams of finite type, it follows that $\Delta$ is of type $H_{3}$ or $I_{2}(5)$. Furthermore, the valency of $a$ in $\Delta^{\prime}$ is at most 2, since no diagram of finite type has a vertex of valency greater than 2 as well as an edge label greater than 3 . So $\operatorname{val}(a) \leq 3$. 
If $\Delta$ is of type $H_{3}$ then $\Delta^{\prime}$ must be of type $H_{4}$, and the valency of $a$ in $\Delta^{\prime}$ is 1 . So $a$ is adjacent to $b$ in $\Gamma$, and $\operatorname{val}(a)=2$. Hence $\Gamma_{a}$ has exactly two components (given that it is reducible). One of these is $\Delta^{\prime}$, of type $H_{4}$, and the other must be of type $H_{3}$ since $\Gamma_{b}$ has a component of type $H_{3}$. Thus $\Gamma$ is

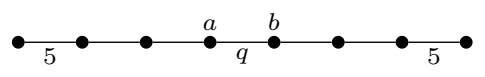

and we see that there is a symmetry interchanging $a$ and $b$.

If $\Delta$ is of type $I_{2}(5)$ then $\Delta^{\prime}$ is of type $H_{3}$ or $H_{4}$. In the former case $a$ has valency 1 in $\Delta^{\prime}$; so $a$ is adjacent to $b$ and $\operatorname{val}(a)=2$. Thus $\Gamma_{a}$ and $\Gamma_{b}$ are of type $I_{2}(5) \times H_{3}$, whence $\Gamma$ is

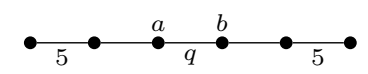

and there is a symmetry interchanging $a$ and $b$. Turning to the other case, observe that the valency of $a$ in $\Delta^{\prime}$ (of type $H_{4}$ ) is 2 , since deleting $a$ gives a component of type $I_{2}(5$ ). If $a$ and $b$ are not adjacent then $\operatorname{val}(a)=2$, and $\Gamma_{a}$ has two components, which must be of types $I_{2}(5)$ and $H_{4}$. So $\Gamma$ is

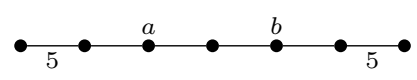

which has a symmetry swapping $a$ and $b$. So suppose that $a$ and $b$ are adjacent, so that $\operatorname{val}(a)=\operatorname{val}(b)=3$. Let $c$ be the end vertex of $\Delta^{\prime}$ adjacent to $a$. If $c$ is also adjacent to $b$ then $\Gamma_{a}$ has only two components, and they are of types $I_{2}(5)$ and $H_{4}$. Furthermore, the valency of $c$ in $\Gamma_{b}$ is 1 ; so $\operatorname{val}(c)=2$, and $\Gamma$ must be

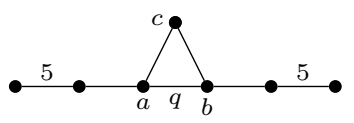

which has a symmetry swapping $a$ and $b$. Finally, suppose that $c$ is not adjacent to $b$. Then $\Gamma_{a}$ and $\Gamma_{b}$ are of type $I_{2}(5) \times A_{1} \times H_{4}$, and there is a $c^{\prime}$ adjacent to $b$ that is not adjacent to $a$. In this case $\Gamma$ is

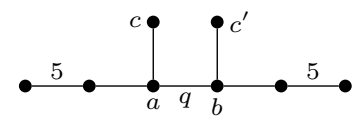

and again there is a symmetry swapping $a$ and $b$.

Next we consider those cases for which $\Gamma_{a}$ and $\Gamma_{b}$ are reducible, and $b$ lies in a component $\Delta$ of $\Gamma_{a}$ of type $H_{3}, H_{4}$ or $I_{2}(m)$ for $m>4$.

Suppose that $\Delta$ has type $H_{3}$. Then the valency of $b$ in $\Gamma_{a}$ is at most 2, and consequently $2 \leq \operatorname{val}(a)=\operatorname{val}(b) \leq 3$. Suppose $\operatorname{val}(a)=\operatorname{val}(b)=3$. Then $a$ and $b$ must be adjacent. The two end vertices of $\Delta$ cannot both be adjacent to $a$, since $\Gamma_{b}$ is reducible. If neither of them are adjacent to $a$ then $\Gamma_{b}$ has three components, and is thus of type $A_{1} \times A_{1} \times H_{3}$. We see that in this case $\Gamma$ is

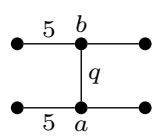

and has a symmetry interchanging $a$ and $b$. If one of the end vertices of $\Delta$ is adjacent to $a$, then $\Gamma_{b}$ has two components and is of type $A_{1} \times H_{3}$. There are four possibilities: two choices for the vertex of $\Delta$ that is adjacent to $a$, and then two choices for the edge incident with $a$ that has the label 5 .
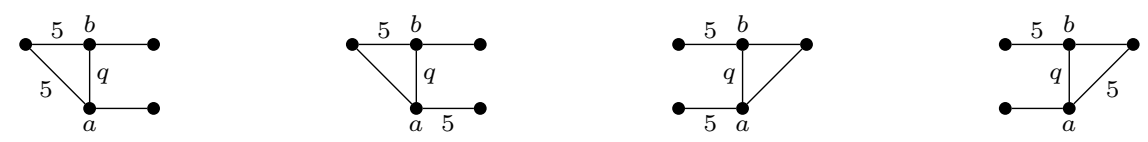
The first and third of these have symmetries interchanging $a$ and $b$, while the other two are both of type $X(q)$.

Now suppose that $\operatorname{val}(a)=\operatorname{val}(b)=2$, still in the case that $\Delta$ is of type $H_{3}$. Note that $\Gamma_{a}$ and $\Gamma_{b}$ must have two components. If $b$ is adjacent to $a$ then it must be an end vertex of $\Delta$, and $\Gamma_{b}$ must be of type $I_{2}(5) \times H_{3}$ or of type $A_{2} \times H_{3}$. The two possibilities for $\Gamma$ are as follows.
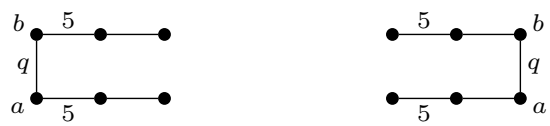

In both cases there is a symmetry interchanging $a$ and $b$. If $b$ is not adjacent to $a$ then it is the middle vertex of $\Delta$, and, since only one of the end vertices can be adjacent to $a$ (given that $\Gamma_{b}$ is not irreducible), we see that the one that is not adjacent to $a$ constitutes a component of $\Gamma_{b}$ of type $A_{1}$. So $\Gamma_{a}$ and $\Gamma_{b}$ are of type $A_{1} \times H_{3}$. The four possibilities for $\Gamma$ are as follows.
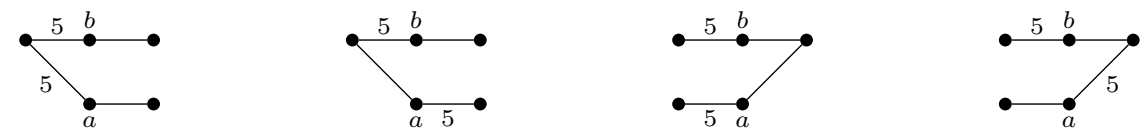

The first and third of these have symmetries interchanging $a$ and $b$, while the other two are both of type $X(2)$.

We have dealt with all possible cases for which $\Delta$ is of type $H_{3}$. Suppose now that $\Delta$ is of type $H_{4}$. As in the $H_{3}$ case we have $2 \leq \operatorname{val}(a)=\operatorname{val}(b) \leq 3$.

Suppose first that $\operatorname{val}(a)=\operatorname{val}(b)=3$. Then $a$ and $b$ are adjacent, and $b$ is not an end vertex of $\Delta$. If no other vertex of $\Delta$ is adjacent to $a$, then $\Gamma_{b}$ has three components and is of type $A_{1} \times A_{2} \times H_{4}$ or $A_{1} \times I_{2}(5) \times H_{4}$. Since $a$ may be either of the inner vertices of of the $H_{4}$ there are potentially four possibilities, but only two of these give $\Gamma_{a}$ isomorphic to $\Gamma_{b}$. The two possibilities for $\Gamma$ are
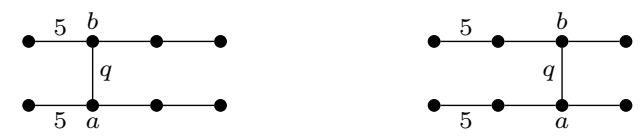

and there is a symmetry interchanging $a$ and $b$. If there were two vertices of $\Delta \backslash\{b\}$ adjacent to $a$ then these vertices could not be adjacent to each other, since if they were then $\Gamma_{b}$ would contain a triangle, contradicting the fact that it is of finite type. So the two components of $\Delta \backslash\{b\}$ would have to each contain one of these vertices, and this is also impossible since then $\Gamma_{b}$ would be irreducible. So it remains to consider the cases in which $a$ is adjacent to exactly one of the vertices in $\Delta \backslash\{b\}$. In each case $\Gamma_{b}$ must have exactly two components, one of which is a component $\Delta \backslash\{b\}$ and the other of which has type $H_{4}$. Now $b$ may be either of the two inner vertices of $\Delta$, and $a$ may be joined to any of the three vertices of $\Delta \backslash\{b\}$. Each of the six choices gives a unique possibility for $\Gamma$.

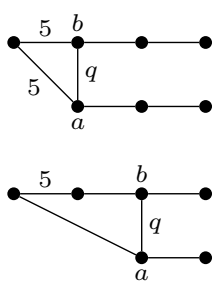

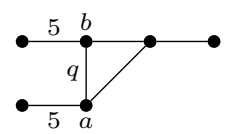

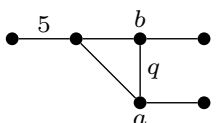

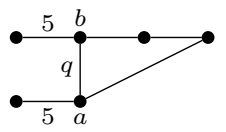

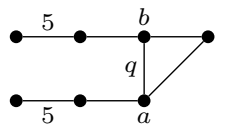

In each case there is a symmetry of $\Gamma$ interchanging $a$ and $b$.

Now suppose that $\operatorname{val}(a)=\operatorname{val}(b)=2$, still in that case that $\Delta$ is of type $H_{4}$. Observe that $\Gamma_{a}$ and $\Gamma_{b}$ have two components. If $b$ is an end vertex of $\Delta$ then it must be adjacent to $a$, and $\Gamma_{b}$ must be either of type $A_{3} \times H_{4}$ or of type $H_{3} \times H_{4}$. The corresponding two 
possibilities for $\Gamma$ are
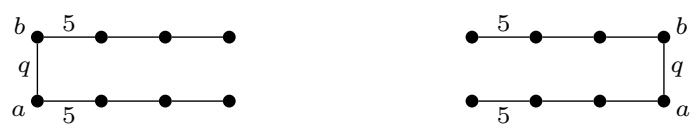

and in both cases there is a symmetry interchanging $a$ and $b$. If $b$ is not adjacent to $a$ then it is an inner vertex of $\Delta$, and, since only one of the other vertices can be adjacent to $a$ (given that $\Gamma_{b}$ is not irreducible and does not contain a triangle), we again obtain six possibilities: $b$ can be either inner vertex of $\Delta$ and $a$ can be adjacent to any of the three vertices of $\Delta \backslash\{b\}$.
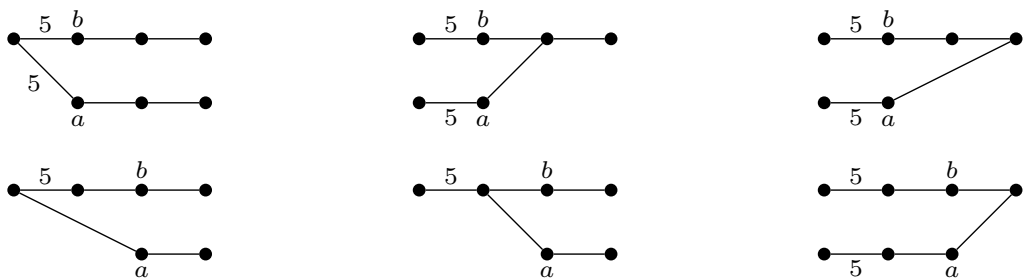

In each case there is a symmetry interchanging $a$ and $b$.

Having dealt with all possible cases for which $\Delta$ is of type $H_{3}$ or $H_{4}$, we assume now that $\Delta$ is of type $I_{2}(m)$. In this case $b$ has valency 1 in in $\Gamma_{a}$, and hence has valency 2 in $\Gamma$. We see that $a$ and $b$ are adjacent, and $a$ is not adjacent to the other vertex of $\Delta$ since $\Gamma_{b}$ is reducible. So $\Gamma_{a}$ and $\Gamma_{b}$ are of type $A_{1} \times I_{2}(m)$. Thus $\Gamma$ is

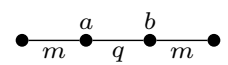

and there is a symmetry swapping $a$ and $b$.

We have now dealt with all cases in which $\Gamma_{a}$ and $\Gamma_{b}$ are reducible, and it remains to deal with the possibility that they are irreducible of type $I_{2}(m), H_{3}$ or $H_{4}$. Observe that $\Gamma$ has three vertices in the first case, four in the second and five in the third.

If $\Gamma_{a}$ is of type $I_{2}(m)$, with vertices $b$ and $c$, then $\Gamma_{b}$ has vertices $a$ and $c$, which must be joined by an edge labelled $m$. So $\Gamma$ is

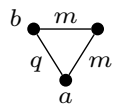

where $q=2$ is allowed. There is a symmetry of the desired kind.

If $\Gamma_{a}$ is of type $H_{3}$ and $b$ is the middle vertex, then $a$ must also be adjacent to the other two vertices, since $\operatorname{val}(a)=\operatorname{val}(b)$. One of these edges must be labelled 5 and the other 3 , since $\Gamma_{b}$ is of type $\mathrm{H}_{3}$. So there are two possibilities for $\Gamma$,
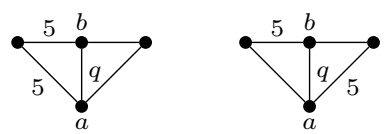

again allowing $q=2$. In each case there is a symmetry swapping $a$ and $b$. If $b$ is an end vertex of $\Gamma_{a}$ then $a$ must be adjacent to exactly one of the remaining two vertices, and since there are two choices for $b$ there are four possibilities for $\Gamma$.
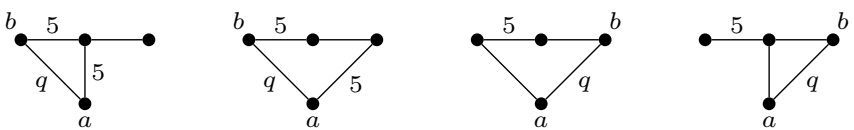

Here again we allow $q=2$, and again each of the diagrams has a symmetry swapping $a$ and $b$.

Finally suppose that $\Gamma_{a}$ is of type $H_{4}$. If $b$ is an inner vertex of $\Gamma_{a}$ then $a$ must be adjacent to exactly two of the other vertices of $\Gamma_{a}$ to ensure that $\operatorname{val}(a)=\operatorname{val}(b)$. Furthermore, 
these two must not belong to the same component of $\Gamma_{a} \backslash\{b\}$ since $\Gamma_{b}$ must not contain a triangle. There are two possibilities for $b$, and then two possibilities for the vertices of $\Gamma_{a} \backslash\{b\}$ adjacent to $a$. The four possibilities for $\Gamma$ are
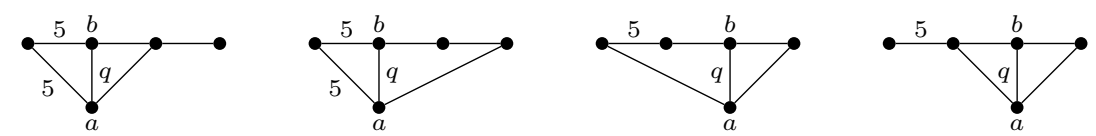

allowing $q=2$. In each case there is a symmetry swapping $a$ and $b$. We are left to consider the cases when $b$ is an end vertex of $\Gamma_{a}$. Suppose first that $b$ at the end with the edge labelled 5. Since $\operatorname{val}(a)=\operatorname{val}(b)$ we see that $a$, like $b$, is adjacent to exactly one of the the other three vertices of $\Gamma_{a}$. It cannot be the middle one, or this would have valency 3 in $\Gamma_{b}$, contrary to the requirement that $\Gamma_{b}$ is of type $H_{4}$. The other two are both possible. If $b$ is at the end of $\Gamma_{a}$ that does not have the edge labelled 5, we again deduce that $a$ must be adjacent to exactly one of the other three vertices of $\Gamma_{a}$. However, only one of these three choices satisfies the requirement that $\Gamma_{b}$ is of type $H_{4}$. So altogether we have three more possibilities for $\Gamma$. They are
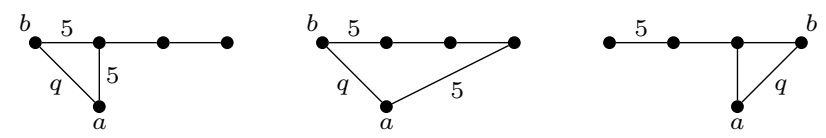

allowing $q=2$. In each case there is a symmetry swapping $a$ and $b$.

\section{COMPletion OF THE PROOF OF THE MAIN THEOREM}

Recall first the following trivial fact.

Lemma 27. Let $W$ be a Coxeter group, and $a, b \in \Pi$. If $m_{a b}$ is odd then $r_{a}$ and $r_{b}$ are conjugate in $W$.

Proof. If $m_{a b}=2 k+1$ then $r_{b}=\left(r_{a} r_{b}\right)^{k} r_{a}\left(r_{a} r_{b}\right)^{-k}$.

Our main theorem is as follows.

Theorem 28. If $W$ is an irreducible non-degenerate nearly finite Coxeter group with finite edge labels, then any automorphism of $W$ that preserves reflections is inner by graph.

Proof. Let $\alpha \in R(W)$, and let $a \in \Pi$ be such that $J=\Pi \backslash\{a\}$ is of finite type. Then $\alpha\left(W_{J}\right)$ is a maximal finite subgroup of $W$, and so equals $t W_{K} t^{-1}$ for some $t \in W$ and some $K \subseteq \Pi$. Replacing $\alpha$ by $w \mapsto t \alpha(w) t^{-1}$ permits us to assume that $\alpha\left(W_{J}\right)=W_{K}$. By Proposition 25 and the fact that $\alpha$ preserves reflections, $W_{J}$ and $W_{K}$ are of the same type. Thus $K=\Pi \backslash\{b\}$ for some $b \in \Pi$ (possibly equal to $a$ ).

Suppose that $J$ does not contain any component of type $H_{3}, H_{4}$ or $I_{2}(m)$ for $m>4$. As $W_{J}$ and $W_{K}$ are of the same type there is an isomorphism $\beta: W_{J} \rightarrow W_{K}$ taking simple reflections to simple reflections. Applying Corollary 19 to the automorphism of $W_{J}$ given by $w \mapsto \beta^{-1}(\alpha(w))$, we deduce that there exists $t \in W_{J}$ such that $\gamma: w \mapsto t\left(\beta^{-1}(\alpha(w))\right) t^{-1}$ is a graph automorphism of $W_{J}$. Thus $\beta \gamma$ is an isomorphism $W_{J} \rightarrow W_{K}$ that takes simple reflections to simple reflections. But $\beta \gamma$ is the restriction to $W_{J}$ of the automorphism $w \mapsto \beta(t) \alpha(w) \beta(t)^{-1}$, and it follows from Theorem 23 that this automorphism is inner by graph. Hence $\alpha$ is inner by graph.

Suppose, on the other hand, that $J$ has a component of type $H_{3}, H_{4}$ or $I_{2}(m)$ for $m>4$. If $W$ is not of type $X(q)$ then by Proposition 26 there is a graph automorphism $\gamma$ of $W$ that takes $W_{K}$ to $W_{J}$. Now $\gamma \alpha$ preserves $W_{J}$, and so Theorem 24 tells us that $\gamma \alpha$ is inner by graph. Hence $\alpha$ is inner by graph. 
It remains to consider that possibility that $W$ is of type $X(q)$ for some $q \geq 2$ and $J \neq K$. Let $\Pi=\{a, b, c, d, e\}$, the Coxeter diagram being as follows.

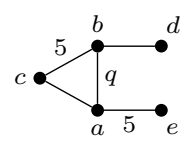

Note that $q=2$ is allowed.

The simple reflection $r_{e}$ is central in $W_{J}$; therefore

$$
\alpha\left(r_{e}\right) \in Z\left(W_{K}\right)=\left\langle r_{d}, w_{K}\right\rangle
$$

where $w_{K}$ is the longest element in $W_{K}$. As $\alpha\left(r_{e}\right)$ is a reflection and $r_{d}$ is the only reflection in $Z\left(W_{K}\right)$ we deduce that $\alpha\left(r_{e}\right)=r_{d}$. Now by Lemma 13

$$
\begin{aligned}
& \operatorname{Ref}\left(W_{J}\right)=\operatorname{Ref}\left(W_{\{c, b, d\}}\right) \cup\left\{r_{e}\right\} \\
& \operatorname{Ref}\left(W_{K}\right)=\operatorname{Ref}\left(W_{\{c, a, e\}}\right) \cup\left\{r_{d}\right\},
\end{aligned}
$$

and it follows that $\alpha\left(\operatorname{Ref}\left(W_{\{c, b, d\}}\right)\right)=\operatorname{Ref}\left(W_{\{c, a, e\}}\right)$. So $\alpha\left(W_{\{c, b, d\}}\right)=W_{\{c, a, e\}}$.

Since the group $W_{\{c, a, e\}}$ has only one conjugacy class of reflections (by Lemma 27), replacing $\alpha$ by $w \mapsto t(\alpha(w)) t^{-1}$ for a suitably chosen $t \in W_{\{c, a, e\}}$ allows us to assume that $\alpha\left(r_{d}\right)=r_{c}$. Now $r=\alpha\left(r_{b}\right)$ has the property that the order of $r r_{c}$ is three (since $r_{b} r_{d}$ has order three), and of the fifteen reflections in $W_{\{c, a, e\}}$ only four satisfy this requirement. Furthermore, these four are permuted transitively by the group $W_{\{a, c\}}$; so again replacing replacing $\alpha$ by $w \mapsto t(\alpha(w)) t^{-1}$ for a suitably chosen $t$ permits us to assume that $r=r_{a}$.

Since $r_{c}$ commutes with $r_{d}$ we deduce that $\alpha\left(r_{c}\right)$ is a reflection that commutes with $r_{c}$. There are just two possibilities for this: the reflection along $e$ and the reflection along $g=-(\lambda+1) e-2 \lambda a-\lambda c$, where $\lambda=2 \cos (\pi / 5)$ (the positive solution of $\left.\lambda^{2}=\lambda+1\right)$. If $\alpha\left(r_{c}\right)=r_{e}$ then Theorem 24 tells us that $\alpha$ is inner by graph. So assume that $\alpha\left(r_{c}\right)=r_{g}$, and let $\alpha\left(r_{a}\right)=r_{f}$, where $f \in \Phi$. We now have

$$
\begin{array}{lll}
\alpha\left(r_{a}\right)=r_{f} & \alpha\left(r_{b}\right)=r_{a} \\
\alpha\left(r_{c}\right)=r_{g} & \alpha\left(r_{d}\right)=r_{c} \\
\alpha\left(r_{e}\right)=r_{d} . &
\end{array}
$$

Since $r_{f} r_{g}$ has order three, $f \cdot g= \pm 1 / 2$. Replacing $f$ by $-f$ if necessary, we may assume that $f \cdot g=-1 / 2$. Since $r_{f} r_{c}$ has order two, $f \cdot c=0$. Since $r_{f} r_{a}$ has order $q$ and $r_{f} r_{d}$ has order five, $f \cdot a=\cos (j \pi / q)$ for some $j$ coprime to $q$ and $f \cdot d=-\cos (k \pi / 5)$ for some $k$ coprime to 5. Let us write $\theta_{a}=\cos (j \pi / q)=f \cdot a$ and $\xi_{a}=\cos (\pi / q)$; note that $\left|\theta_{a}\right| \leq \xi_{a}$. Let us also write $\theta_{d}=\cos (k \pi / 5)=-f \cdot d$; note that $\left|\theta_{d}\right| \leq \cos (\pi / 5)=\lambda / 2$.

For later reference, note that $a \cdot g=\frac{1}{2}(1-\lambda)$, while $c \cdot g=e \cdot g=d \cdot g=0$.

Define $x=\lambda a+\frac{1}{2} \lambda c-\theta_{d} d+\left(\frac{3}{2}+(2-2 \lambda) \theta_{a}\right) e$. Note that $x \in V_{K}$. We compute $x \cdot v$ for each $v$ in the basis $\{a, c, d, g\}$ of $V_{K}$.

$$
\begin{aligned}
x \cdot a & =\lambda-\frac{1}{4} \lambda+0+\left(\frac{3}{2}+(2-2 \lambda) \theta_{a}\right)\left(-\frac{1}{2} \lambda\right) \\
& =\frac{3}{4} \lambda-\frac{3}{4} \lambda+\left(\lambda^{2}-\lambda\right) \theta_{a} \\
& =\theta_{a} \\
& =f \cdot a, \\
x \cdot c & =-\frac{1}{2} \lambda+\frac{1}{2} \lambda+0+0 \\
& =0 \\
& =f \cdot c, \\
x \cdot d & =0+0-\theta_{d}+0 \\
& =f \cdot d,
\end{aligned}
$$




$$
\begin{aligned}
x \cdot g & =\frac{1}{2} \lambda(1-\lambda)+0+0+0 \\
& =-\frac{1}{2} \\
& =f \cdot g .
\end{aligned}
$$

Thus $x$ is the orthogonal projection of $f$ onto $V_{K}$.

Now define

$y=\left((1+2 \lambda)+(4+4 \lambda) \xi_{a}\right) a+\left(\left(\frac{1}{2}+\frac{3}{2} \lambda\right)+(2+2 \lambda) \xi_{a}\right) c+\frac{1}{2} d+\left(\left(1+\frac{3}{2} \lambda\right)+(2+4 \lambda) \xi_{a}\right) e$.

We find that

$$
\begin{aligned}
y \cdot a & =(1+2 \lambda)+(4+4 \lambda) \xi_{a}-\frac{1}{2}\left(\left(\frac{1}{2}+\frac{3}{2} \lambda\right)+(2+2 \lambda) \xi_{a}\right)+0 \\
& \quad-\frac{1}{2} \lambda\left(\left(1+\frac{3}{2} \lambda\right)+(2+4 \lambda) \xi_{a}\right) \\
& =\frac{3}{4}+\frac{3}{4} \lambda-\frac{3}{4} \lambda^{2}+\left(3+2 \lambda-2 \lambda^{2}\right) \xi_{a} \\
& =\xi_{a}=-b \cdot a, \\
y \cdot c & =-\frac{1}{2}\left((1+2 \lambda)+(4+4 \lambda) \xi_{a}\right)+\left(\left(\frac{1}{2}+\frac{3}{2} \lambda\right)+(2+2 \lambda) \xi_{a}\right)+0+0 \\
& =\frac{1}{2} \lambda=-b \cdot c \\
y \cdot d & =\frac{1}{2}=-b \cdot d, \\
y \cdot e & =-\frac{1}{2} \lambda\left((1+2 \lambda)+(4+4 \lambda) \xi_{a}\right)+0+0+\left(\left(1+\frac{3}{2} \lambda\right)+(2+4 \lambda) \xi_{a}\right) \\
& =1+\lambda-\lambda^{2}+\left(2+2 \lambda-2 \lambda^{2}\right) \xi_{a} \\
& =0=-b \cdot e .
\end{aligned}
$$

Thus $y$ is the projection of $-b$ onto $V_{K}$. Put $u=b+y$, a nonzero element of the orthogonal complement of $V_{K}$. We can confirm that $W$ is nondegenerate by checking that $u \cdot u<0$. Indeed,

$$
\begin{aligned}
u \cdot u & =(y+b) \cdot u=b \cdot u=1+b \cdot y \\
& =1-\xi_{a}\left((1+2 \lambda)+(4+4 \lambda) \xi_{a}\right)-\frac{1}{2} \lambda\left(\left(\frac{1}{2}+\frac{3}{2} \lambda\right)+(2+2 \lambda) \xi_{a}\right)-\frac{1}{4} \\
& =-\lambda-2(1+2 \lambda) \xi_{a}-(4+4 \lambda) \xi_{a}^{2} \\
& <0,
\end{aligned}
$$

as expected. Now we find that

$$
x \cdot e=-\frac{1}{2} \lambda^{2}+0+0+\frac{3}{2}+(2-2 \lambda) \theta_{a}=1-\frac{1}{2} \lambda+(2-2 \lambda) \theta_{a}
$$

and thus

$$
\begin{aligned}
x \cdot x & =\lambda a \cdot x+\frac{1}{2} \lambda c \cdot x-\theta_{d} d \cdot x+\left(\frac{3}{2}+(2-2 \lambda) \theta_{a}\right) e \cdot x \\
& =\lambda \theta_{a}+0+\theta_{d}^{2}+\left(\frac{3}{2}+(2-2 \lambda) \theta_{a}\right)\left(1-\frac{1}{2} \lambda+(2-2 \lambda) \theta_{a}\right) \\
& =\theta_{d}^{2}+\frac{3}{2}-\frac{3}{4} \lambda+\left(\lambda+3(1-\lambda)+\left(3-3 \lambda+\lambda^{2}\right)\right) \theta_{a}+4(1-\lambda)^{2} \theta_{a}^{2} \\
& =\theta_{d}^{2}+\frac{3}{2}-\frac{3}{4} \lambda+2(3-2 \lambda) \theta_{a}+4(2-\lambda) \theta_{a}^{2} .
\end{aligned}
$$

Note that $\theta_{d}^{2} \leq \frac{1}{4} \lambda^{2}=\frac{1}{4}(1+\lambda)$. Furthermore, since we also have that $\left|\theta_{a}\right| \leq \xi_{a}$,

$$
\begin{aligned}
x \cdot x & \leq \frac{7}{4}-\frac{1}{2} \lambda+2|3-2 \lambda|\left|\theta_{a}\right|+4|2-\lambda|\left|\theta_{a}\right|^{2} \\
& \leq \frac{7}{4}-\frac{1}{2} \lambda+2(2 \lambda-3) \xi_{a}+4(2-\lambda) \xi_{a}^{2},
\end{aligned}
$$

and therefore

$$
\begin{aligned}
1-x \cdot x-u \cdot u & \geq 1-\frac{7}{4}+\frac{1}{2} \lambda-2(2 \lambda-3) \xi_{a}-4(2-\lambda) \xi_{a}^{2} \\
& +\lambda+2(1+2 \lambda) \xi_{a}+(4+4 \lambda) \xi_{a}^{2} \\
= & \left(\frac{3}{2} \lambda-\frac{3}{4}\right)+8 \xi_{a}+(8 \lambda-4) \xi_{a}^{2},
\end{aligned}
$$


which is clearly positive since all the terms are positive. Therefore

$$
\frac{1-x \cdot x}{u \cdot u}<1
$$

as $u \cdot u<0$.

As in our earlier proofs we write $f=x+\omega u$, and use Lemma 3 to deduce that either $\omega \geq 1$ (if $x \in \Phi^{+}$) or $\omega \leq-1$ (if $x \in \Phi^{-}$), and hence that $\omega^{2} \geq 1$. Since $f$ is a root,

$$
\begin{aligned}
1 & =f \cdot f \\
& =(x+\omega u) \cdot(x+\omega u) \\
& =x \cdot x+\omega^{2} u \cdot u,
\end{aligned}
$$

and hence

$$
\omega^{2}=\frac{1-x \cdot x}{u \cdot u}<1
$$

and this is a contradiction. So this case cannot arise, and we conclude that $\alpha$ is inner by graph.

The next result provides a strengthening of our main theorem.

Theorem 29. Theorem 28 remains valid if the assumption that $W$ is nondegenerate is omitted.

Proof. Let $n$ be the rank of $W$. Since $W$ is nearly finite there is a subspace of $V$ of dimension $n-1$ on which the bilinear form $B$ associated with $W$ is positive definite. If $B$ is degenerate then its radical must be complementary to this positive definite subspace, and so $B$ is positive semidefinite with a 1-dimensional radical. The classification of irreducible positive semidefinite Coxeter groups is given in [11, Section 2.7]; the groups concerned are isomorphic to the affine Weyl groups, and correspond to the following list of Coxeter diagrams.
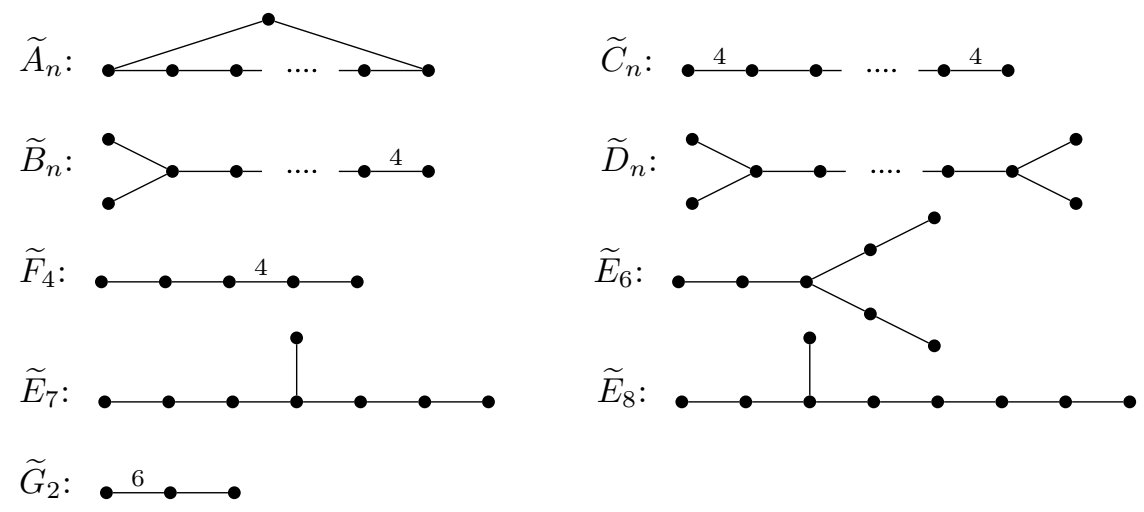

In each case the rank is one greater than the name might suggest: for example, $\widetilde{A}_{n}$ has rank $n+1$. For types $\widetilde{C}_{n}$ and $\widetilde{A}_{n}$ we require $n \geq 2$; type $\widetilde{A}_{1}$ is not covered by the present theorem since its diagram has $\infty$ as an edge label (although the conclusion of the theorem in fact remains valid). For $\widetilde{B}_{n}$ and $\widetilde{D}_{n}$ we require $n \geq 3$ and $n \geq 4$ respectively.

For all cases except $\widetilde{A}_{n}$ the desired conclusion that every reflection-preserving automorphism is inner by graph follows immediately from Corollary 19 . So suppose that $W$ is of type $\widetilde{A}_{n}$, and let $\alpha \in R(W)$. Choose a function $\phi_{\alpha}: \Pi \rightarrow \Phi$ such that $\alpha\left(r_{a}\right)=r_{\phi_{\alpha}(a)}$ for all $a \in \Pi$. Write $\Pi=\left\{a_{0}, a_{1}, \ldots, a_{n}\right\}$, where $a_{0}$ is adjacent to $a_{n}$ and $a_{i}$ is adjacent to $a_{i-1}$ for $1 \leq i \leq n$. Then $\phi_{\alpha}\left(a_{i}\right) \cdot \phi_{\alpha}\left(a_{j}\right)$ is $\pm 1 / 2$ if $a_{i}$ and $a_{j}$ are adjacent, and zero otherwise. We can successively choose signs $\varepsilon_{1}, \varepsilon_{2}, \ldots, \varepsilon_{n}$ so that when $\phi_{\alpha}\left(a_{i}\right)$ is replaced by $\varepsilon_{i} \phi_{\alpha}\left(a_{i}\right)$ we have $\phi_{\alpha}\left(a_{i-1}\right) \cdot \phi_{\alpha}\left(a_{i}\right)=-1 / 2$ for $1 \leq i \leq n$. Now if $\phi_{\alpha}\left(a_{0}\right) \cdot \phi_{\alpha}\left(a_{n}\right)=-1 / 2$ 
then Theorem 18 guarantees that $\alpha$ is inner by graph. But if $\phi_{\alpha}\left(a_{0}\right) \cdot \phi_{\alpha}\left(a_{n}\right)=1 / 2$ then it is readily checked that the matrix whose $(i, j)$ entry is $\phi_{\alpha}\left(a_{i}\right) \cdot \phi_{\alpha}\left(a_{j}\right)$ is positive definite, contradicting the fact that $B$ is degenerate.

\section{GROUPS WITH A FINITE IRREDUCIBLE MAXIMAL PARABOLIC SUBGROUP}

In this section we shall not assume that the automorphism $\alpha$ preserves reflections; instead we shall prove that it must preserve reflections, given appropriate extra hypotheses. Specifically, we shall investigate nearly finite Coxeter groups with a finite irreducible maximal parabolic subgroup.

Our analysis depends upon some facts concerning automorphism groups of finite irreducible Coxeter groups. We proceed to give a brief discussion of this topic.

Let $W$ be a finite irreducible Coxeter group. The centre of $W$ is either trivial or of order two. We denote the nonidentity element of the centre by $z$, when it exists. In all of these cases, $z$ is equal to $w_{\Pi}$, the longest element of $W$. The group of all homomorphisms from $W$ to the cyclic group of order two is isomorphic to the abelianization of $W$, and has order four if the Coxeter diagram has an even edge label, and order two otherwise. Let $\mathscr{H}$ denote the group of all homomorphisms from $W$ to its centre. It is clear that for all $f \in \mathscr{H}$ the mapping $\alpha_{f}: w \mapsto w f(w)$ is a homomorphism from $W$ to itself, and is an automorphism precisely when $z$ is in the kernel of $f$ (so that $z f(z) \neq 1$ ). These automorphisms are reflection preserving if and only if $W$ is of rank 2. Moreover, $\alpha_{f g}=\alpha_{f} \alpha_{g}$ whenever $f(z)=g(z)=1$; hence $\mathscr{A}(W)=\left\{\alpha_{f} \mid z \in \operatorname{ker} f\right\}$ is a subgroup of Aut $(W)$. Clearly all elements of $\mathscr{A}(W)$ are self-inverse.

If $W$ is of type $B_{n}$, with the following diagram,

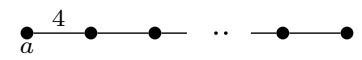

we let $\zeta: W \rightarrow Z(W)$ be the homomorphism that maps $r_{a} \mapsto 1$ and all other simple reflections to $z$. It is easily checked that $\zeta(z)=1$, and so $\alpha_{\zeta} \in \operatorname{Aut}(W)$. Similarly, for type $F_{4}$ there are two conjugacy classes of reflections, and we let $\zeta: W \rightarrow Z(W)$ map the reflections in one of these to $z$ and those in the other to 1. Again $\alpha_{\zeta} \in \operatorname{Aut}(W)$. In all cases where $w_{\Pi}=z \in Z(W)$, let $\xi: W \rightarrow Z(W)$ be the homomorphism that maps each simple reflection to $z$. Then $\xi(w)=z^{l(w)}$ for all $w \in W$, and so $\xi(z)=1$ precisely when $l(z)$ is even. In particular, $\alpha_{\xi} \in \operatorname{Aut}(W)$ when $W$ is of type $B_{2 k}, D_{2 k}, E_{8}, F_{4}$ or $H_{4}$. A straightforward calculation shows that $\alpha_{\xi}$ commutes with all reflection preserving automorphisms.

Proposition 30. The group $\mathscr{A}(W)$ defined above is trivial if $W$ is of type $A_{n}, D_{2 k+1}, E_{6}$, $E_{7}$ or $H_{3}$, has order two if $W$ is of type $B_{2 k+1}, D_{2 k}, E_{8}$ or $H_{4}$, and has order four if $W$ is of type $B_{2 k}$ or $F_{4}$.

Indeed, $\mathscr{A}(W)=\left\langle\alpha_{\xi}\right\rangle$ for types $D_{2 k}, E_{8}$ and $H_{4}$, while $\mathscr{A}(W)=\left\langle\alpha_{\zeta}\right\rangle$ for $B_{2 k+1}$, and $\mathscr{A}(W)=\left\langle\alpha_{\xi}, \alpha_{\zeta}\right\rangle$ for $B_{2 k}$ and $F_{4}$.

When $W$ is of type $I_{2}(m)$ it is obvious that $\operatorname{Aut}(W)=R(W)$, and in all other cases $\mathscr{A}(W) \cap R(W)$ is trivial. So in these cases $\operatorname{Aut}(W)$ has a subgroup isomorphic to the semidirect product $R(W) \rtimes \mathscr{A}(W)$ (since it is obvious that $R(W)$ is normal in $\operatorname{Aut}(W)$ ). Let $\operatorname{Gr}(W)$ be the group of all graph automorphisms of $W$. By Theorem 18 we know that $R(W)=\operatorname{Inn}(W) \mathrm{Gr}(W)$ unless $W$ is of type $H_{3}$ or $H_{4}$. In these cases there are at most two possibilities for the function $\phi_{\alpha}$ in Lemma 15 , and so $[R(W): \operatorname{Inn}(W) \operatorname{Gr}(W)] \leq 2$. In fact, as we shall see in the proof of Proposition 32. types $H_{3}$ and $H_{4}$ do possess reflection preserving automorphisms that are not inner by graph; so $[R(W): \operatorname{Inn}(W) \operatorname{Gr}(W)]=2$ in each case. For $W$ of type $I_{2}(m)$ it can be checked that $R(W) / \operatorname{Inn}(W) \operatorname{Gr}(W)$ is isomorphic to the group of units of the ring of integers modulo $m$.

As is well known, the groups of type $A_{n}$ are isomorphic to the finite symmetric groups, and all automorphisms are inner except when $n=5$, in which case $\operatorname{Inn}(W)=R(W)$ has 
index two in $\operatorname{Aut}(W)$. The main assertion of Theorem 31 below is that there are no other finite irreducible Coxeter groups $W$ such that $\operatorname{Aut}(W) \neq R(W) \mathscr{A}(W)$.

Whenever the group of symmetries of the Coxeter diagram has order 2, we let $\gamma$ be the corresponding nontrivial graph automorphism of $W$. If $W$ is of type $H_{3}$ or $H_{4}$ we let $\rho$ be the non-inner reflection preserving automorphism constructed in the proof of Proposition 32 below. The following theorem then describes the classification of automorphisms of finite irreducible Coxeter groups.

Theorem 31. If $W$ is a Coxeter group of type $B_{n}, D_{n}, E_{6}, E_{7}, E_{8}, F_{4}, H_{3}$ or $H_{4}$ then $\operatorname{Aut}(W)=R(W) \mathscr{A}(W)$. Specifically:

(1) If $W$ is of type $B_{n}$, $n$ odd, then $\operatorname{Aut}(W) \cong W /\left\langle w_{\Pi}\right\rangle \rtimes\left\langle\alpha_{\zeta}\right\rangle$.

(2) If $W$ is of type $B_{n}$, neven, then $\operatorname{Aut}(W) \cong\left(\left(W /\left\langle w_{\Pi}\right\rangle\right) \rtimes\left\langle\alpha_{\zeta}\right\rangle\right) \times\left\langle\alpha_{\xi}\right\rangle$.

(3) If $W$ is of type $D_{n}, n$ odd, then $\operatorname{Aut}(W)=R(W) \cong W$.

(4) If $W$ is of type $D_{n}$, $n$ even and $n>4$, then $\operatorname{Aut}(W) \cong\left(\left(W /\left\langle w_{\Pi}\right\rangle\right) \rtimes\langle\gamma\rangle\right) \times\left\langle\alpha_{\xi}\right\rangle$.

(5) If $W$ is of type $D_{4}$ then $\operatorname{Aut}(W) \cong\left(\left(W /\left\langle w_{\Pi}\right\rangle\right) \rtimes \operatorname{Sym}_{3}\right) \times\left\langle\alpha_{\xi}\right\rangle$.

(6) If $W$ is of type $E_{6}$ then $\operatorname{Aut}(W)=R(W) \cong W$.

(7) If $W$ is of type $E_{7}$ then $\operatorname{Aut}(W)=R(W) \cong W /\left\langle w_{\Pi}\right\rangle$.

(8) If $W$ is of type $E_{8}$ then $\operatorname{Aut}(W) \cong\left(W /\left\langle w_{\Pi}\right\rangle\right) \times\left\langle\alpha_{\xi}\right\rangle$.

(9) If $W$ is of type $F_{4}$ then $\operatorname{Aut}(W) \cong\left(W /\left\langle w_{\Pi}\right\rangle\right) \rtimes\left\langle\gamma, \alpha_{\chi}\right\rangle$.

(10) If $W$ is of type $H_{3}$ then $\operatorname{Aut}(W) \cong\left(W /\left\langle w_{\Pi}\right\rangle\right) \rtimes\langle\rho\rangle$.

(11) If $W$ is of type $H_{4}$ then $\operatorname{Aut}(W) \cong\left(\left(W /\left\langle w_{\Pi}\right\rangle\right) \rtimes\langle\rho\rangle\right) \times\left\langle\alpha_{\xi}\right\rangle$.

Proof. (Outline) In all cases we consider the sizes of the conjugacy classes of involutions; see [3]. We consider the simpler cases first.

For type $E_{6}$ there are 4 classes of involutions, of sizes $270,540,45$ and 36 , the class of reflections being the one of size 36 . Clearly all automorphisms must preserve reflections and hence are inner by Corollary 19 . In this case the graph automorphism $\gamma$ is inner, being conjugation by $w_{\Pi}$. Thus we have:

$$
\operatorname{Aut}(W)=R(W) \cong W .
$$

For type $E_{7}$ there are 63 reflections, and the other classes of involutions have sizes 945 , $3780,315,3780,316,945,63$ and 1 . In this case $w_{\Pi}$ is central and so if $r$ is a simple reflection then $r w_{\Pi}$ is an involution. Thus the other class of involutions that has size 63 must be the class of $r w_{\Pi}$. But $l\left(r w_{\Pi}\right)=62$ is even, and so this class does not generate $W$. In the absence of graph automorphisms we therefore have:

$$
\operatorname{Aut}(W)=R(W) \cong W /\left\langle w_{\Pi}\right\rangle .
$$

For type $E_{8}$ there are 120 reflections, and the other classes of involutions have sizes 3780, 37800, 113400, 3150, 37800, 3780, 120 and 1. Again the second class of size 120 is the class of $r w_{\Pi}$, where $r$ is a reflection. But $\alpha_{\xi}$ is an automorphism that interchanges these two classes. Thus, up to $\alpha_{\xi}$, automorphisms preserve reflections and hence are inner. We have:

$$
\operatorname{Aut}(W) \cong\left(W /\left\langle w_{\Pi}\right\rangle\right) \times\left\langle\alpha_{\xi}\right\rangle .
$$

For type $\mathrm{H}_{3}$ the class of reflections has size 15 while the other classes have sizes 15 and 1. By an argument similar to that used for type $E_{7}$, the second class of size 15 does not generate $W$. Thus $\operatorname{Aut}(W)=R(W)$. Since $w_{\Pi}$ is central, $\operatorname{Inn}(W) \cong W /\left\langle w_{\Pi}\right\rangle$. But, as explained above, $\operatorname{Inn}(W)$ has index two in $R(W)$ in this case. So we have:

$$
\operatorname{Aut}(W) \cong\left(W /\left\langle w_{\Pi}\right\rangle\right) \rtimes\langle\rho\rangle .
$$

For type $H_{4}$ the class of reflections has size 60 while the other classes have sizes 450 , 60 and 1 . In this case $\alpha_{\xi}$ is an automorphism that swaps the two classes of size 60 , and so $\operatorname{Aut}(W)$ is the product of $R(W)$ and $\left\langle\alpha_{\xi}\right\rangle$. So we have:

$$
\operatorname{Aut}(W) \cong\left(\left(W /\left\langle w_{\Pi}\right\rangle\right) \rtimes\langle\rho\rangle\right) \times\left\langle\alpha_{\xi}\right\rangle .
$$


For type $F_{4}$ there are two classes of reflections, each of size 12, and they are interchanged by the graph automorphism $\gamma$. The remaining classes have sizes $18,77,12,12$ and 1. If $r$ and $s$ are representatives of the classes of reflections then $r w_{\Pi}$ and $s w_{\Pi}$ are representatives of the other classes of size 12 . We can take the homomorphism $\zeta: W \rightarrow Z(W)$ defined above to satisfy $\zeta(r)=w_{\Pi}$ and $\zeta(s)=1$. The reflection subgroup generated by the reflections conjugate to $r$ is of type $D_{4}$, and contains $w_{\Pi}$. Thus classes of $r$ and $r w_{\Pi}$ together do not generate $W$, and the same applies for the classes of $s$ and $s w_{\Pi}$. This leaves 8 possible targets for the images of the two classes of reflections under the action of an automorphism. Since $\gamma$ and $\alpha_{\zeta}$ generate a copy of the dihedral group of order eight, we have:

$$
\operatorname{Aut}(W) \cong\left(W /\left\langle w_{\Pi}\right\rangle\right) \rtimes\left\langle\gamma, \alpha_{\zeta}\right\rangle
$$

For types $B_{n}$ and $D_{n}$ we use the well known fact that groups of these types are isomorphic to $\mathscr{E}_{n} \rtimes \operatorname{Sym}_{n}$ and $\mathscr{E}_{n}^{\prime} \rtimes \operatorname{Sym}_{n}$, where $\mathscr{E}_{n}=\left\langle x_{1}, x_{2}, \ldots, x_{n}\right\rangle$ is an elementary abelian 2-group of order $2^{n}$, and $\mathscr{E}_{n}^{\prime}$ is the subgroup of $\mathscr{E}_{n}$ generated by elements of the form $x_{i} x_{j}$. Thus involutions have the form $\beta_{i} \pi_{j}$ where $\beta_{i}$ is a product of $i$ distinct transpositions and $\pi_{j}$ is a product of $j$ distinct $x_{k}$ 's, with the proviso that if $x_{h}$ appears in $\pi_{j}$ and the transposition ( $h k$ ) appears in $\beta_{i}$ then $x_{k}$ also appears in $\pi_{j}$. It can be shown that if $W$ is of type $B_{n}$ then $\beta_{i} \pi_{j}$ is conjugate to an element $\beta_{i} \pi_{l}^{\prime}$ where no term $x_{k}$ in $\pi_{l}^{\prime}$ is moved by $\beta_{i}$. The same is true in type $D_{n}$ provided that $2 i<n$, although $j$ (and $l$ ) must be even in this case. The number of elements in the class is

$$
\frac{n !}{i ! j !(n-j-2 i) !}
$$

If $n=2 t$ then the involutions in $D_{n}$ of the form $\beta_{t} \pi_{j}$ are conjugate either to $\beta_{t}$ or to $\beta_{t} x_{k} x_{l}$, where $(k l)$ is some transposition in $\beta_{t}$. We obtain two classes of size

$$
\frac{(2 i) !}{2 i !}
$$

In type $B_{n}$ the classes of reflections have representatives $\beta_{1} \pi_{0}$ and $\beta_{0} \pi_{1}$, with sizes $n(n-1)$ and $n$ respectively. The latter class does not occur in type $D_{n}$. The only coincidences of class sizes that involve classes of reflections are as follows.

- In type $B_{4}$ the class of (12)(34) has the same size as that of (12).

- In type $D_{4}$ the class of $(12)(34) x_{1} x_{2}$ has the same size as that of (12).

- In type $B_{8}$ the classes of $x_{1} x_{2} x_{3}$ and $x_{1} x_{2} x_{3} x_{4} x_{5}$ have the same size as that of (12).

- In type $B_{n}$ the class of $x_{1} w_{\Pi}$ has the same size as that of $x_{1}$.

- In types $B_{n}$ and $D_{2 t}$ the class of (12) $w_{\Pi}$ has the same size as that of (12).

The first three cannot give rise to automorphisms as the classes that would contain the images of the reflections do not generate $W$. The same applies in the fourth case when $n$ is odd, while when $n$ is even the automorphism $\alpha_{\xi}$ interchanges the two classes in question. In the fifth case the two classes are interchanged by the automorphism $\alpha_{\zeta}$ of $B_{n}$ or the automorphism $\alpha_{\xi}$ of $D_{2 t}$. Thus in all cases $\operatorname{Aut}(W)$ is generated by $\mathscr{A}(W)$ and $R(W)$. Finally, observing that for $n$ odd the graph automorphism of groups of type $D_{n}$ is induced by conjugation by $w_{\Pi}$ and that the group of graph automorphisms of type $D_{4}$ is isomorphic to $\mathrm{Sym}_{3}$, we have the following conclusions.

If $W$ is of type $B_{n}, n$ odd, then

$$
\operatorname{Aut}(W) \cong W /\left\langle w_{\Pi}\right\rangle \rtimes\left\langle\alpha_{\zeta}\right\rangle .
$$

If $W$ is of type $B_{n}, n$ even, then

$$
\operatorname{Aut}(W) \cong\left(\left(W /\left\langle w_{\Pi}\right\rangle\right) \rtimes\left\langle\alpha_{\zeta}\right\rangle\right) \times\left\langle\alpha_{\xi}\right\rangle .
$$

If $W$ is of type $D_{n}, n$ odd, then

$$
\operatorname{Aut}(W)=R(W) \cong W .
$$


If $W$ is of type $D_{n}, n>4$ even, then

$$
\operatorname{Aut}(W) \cong\left(\left(W /\left\langle w_{\Pi}\right\rangle\right) \rtimes\langle\gamma\rangle\right) \times\left\langle\alpha_{\xi}\right\rangle .
$$

If $W$ is of type $D_{4}$ then

$$
\operatorname{Aut}(W) \cong\left(\left(W /\left\langle w_{\Pi}\right\rangle\right) \rtimes \operatorname{Sym}_{3}\right) \times\left\langle\alpha_{\xi}\right\rangle .
$$

We now use the above discussion to prove the results that we actually need.

Proposition 32. Let $W$ be a finite Coxeter group, and let $\alpha$ be an automorphism of $W$ that preserves reflections. Then $\alpha$ preserves the set of parabolic subgroups of $W$.

Proof. By Lemma 14 we know that $\alpha$ permutes the irreducible components of $W$, and by Proposition 25 it maps each component to a component of the same type. So replacing $\alpha$ by $\gamma \alpha$ for a suitable graph automorphism $\gamma$, we can assume that $\alpha$ preserves each component. So it is sufficient to prove the result for irreducible Coxeter groups. Since the group of all automorphisms that preserve parabolic subgroups contains the inner and graph automorphisms we have only to consider types $H_{3}$ and $H_{4}$, and it is sufficient to prove that one element of $R(W)$ that is not inner by graph preserves the set of parabolic subgroups.

Let $W$ be of type $H_{4}$ and let $\Pi=\{a, b, c, d\}$, with the following diagram:

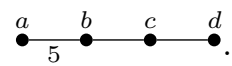

Let $\lambda=\frac{1}{2}(1+\sqrt{5})$, and define $a^{\prime}=(3 \lambda+2) a+(3 \lambda+3) b+2(\lambda+1) c+(\lambda+1) d$. It can be checked that $a^{\prime} \in \Phi$ and $a^{\prime} \cdot c=a^{\prime} \cdot d=0$; furthermore, $a^{\prime} \cdot b=\frac{1}{2}(\lambda-1)=-\cos (2 \pi / 5)$. So there is an automorphism $\rho \in R(W)$ that fixes $r_{b}, r_{c}$ and $r_{d}$ and takes $r_{a}$ to $r_{a^{\prime}}$. If we define

$$
\begin{aligned}
& w_{1}=r_{b} r_{a} r_{c} r_{b} r_{a} r_{b} r_{d} r_{c} r_{b} r_{a} r_{b} r_{a} r_{c} r_{b} r_{a} r_{d} r_{c} r_{b} r_{a}, \\
& w_{2}=r_{c} r_{b} r_{a} r_{b} r_{a} r_{d} r_{c} r_{b} r_{a} r_{b} r_{a} r_{c} r_{b} r_{a} r_{b} r_{d} r_{c} r_{b} r_{a} r_{b} r_{a}, \\
& w_{3}=r_{b} r_{a} r_{b} r_{a} r_{d} r_{c} r_{b} r_{a} r_{b} r_{a} r_{c} r_{b} r_{a} r_{b} r_{d} r_{c} r_{b} r_{a} r_{b} r_{a} r_{c} r_{b} r_{a},
\end{aligned}
$$

then a straightforward calculation reveals that

$$
\begin{aligned}
& \left\{w_{1} a^{\prime}, w_{1} c, w_{1} d\right\}=\{a, d, c\}, \\
& \left\{w_{2} a^{\prime}, w_{2} b, w_{2} d\right\}=\{a+\lambda b, a, d\}, \\
& \left\{w_{3} a^{\prime}, w_{3} b, w_{3} c\right\}=\{c, \lambda a+(\lambda+1) b+c, a\},
\end{aligned}
$$

and therefore

$$
\begin{aligned}
& \rho\left(W_{\{a, c, d\}}\right)=w_{1}^{-1} W_{\left\{w_{1} a^{\prime}, w_{1} c, w_{1} d\right\}} w_{1}=w_{1}^{-1} W_{\{a, d, c\}} w_{1}, \\
& \rho\left(W_{\{a, b, d\}}\right)=w_{2}^{-1} W_{\left\{w_{2} a^{\prime}, w_{2} b, w_{2} d\right\}} w_{2}=w_{2}^{-1} W_{\{a, b, d\}} w_{2}, \\
& \rho\left(W_{\{a, b, c\}}\right)=w_{3}^{-1} W_{\left\{w_{3} a^{\prime}, w_{3} b, w_{3} c\right\}} w_{3}=w_{3}^{-1} W_{\{a, b, c\}} w_{3} .
\end{aligned}
$$

In particular, these are all parabolic subgroups. It is obvious that $\rho\left(W_{\{b, c, d\}}\right)$ is parabolic, and so $\rho$ preserves maximal parabolic subgroups. Since the result is known for groups of lower rank apart from $\mathrm{H}_{3}$, the proof for $\mathrm{H}_{3}$ is all that remains to be done.

So now let $W$ be a group of type $H_{3}$, and let $\Pi=\{a, b, c\}$, arranged as for $H_{4}$. Let $a^{\prime}=(\lambda+1) a+(\lambda+1) b+c$. It is readily checked that $a^{\prime} \in \Phi$, and also that $a^{\prime} \cdot c=\frac{1}{2}(\lambda-1)$ and $a^{\prime} \cdot b=0$. So there is an automorphism $\rho$ that interchanges $r_{b}$ and $r_{c}$ and takes $r_{a}$ to $r_{a^{\prime}}$. If $w=r_{b} r_{a} r_{b} r_{a}$ then $\left\{w a^{\prime}, w b\right\}=\{c, a\}$, and so $\rho\left(W_{\{a, c\}}\right)$ is a parabolic subgroup. If $w=r_{c} r_{b} r_{a}$ then $\left\{w a^{\prime}, w c\right\}=\{\lambda a+b, b\}$, and so $\rho\left(W_{\{a, b\}}\right)$ is a parabolic subgroup. So $\rho$ preserves maximal parabolic subgroups, and hence all parabolic subgroups, since the result is already known for smaller rank. 
Proposition 33. Suppose that $W$ is a finite irreducible Coxeter group of rank at least three, and suppose that $\alpha$ is a nonidentity automorphism in $\mathscr{A}(W)$. If $W^{\prime}$ is a maximal parabolic subgroup of $W$ such that $\alpha\left(W^{\prime}\right)$ is also a parabolic subgroup then $\alpha(w)=w$ for all $w \in W^{\prime}$.

Proof. Let $\alpha=\alpha_{f}$ where $f \in \mathscr{H}$, and let $z$ be the element of $W$ of maximal length. Suppose that $W^{\prime}$ is a maximal parabolic subgroup such that $\alpha(w) \neq w$ for at least one element $w \in W^{\prime}$, and suppose, for a contradiction, that $\alpha\left(W^{\prime}\right)$ is a parabolic subgroup. It is trivial to check that inner automorphisms commute with all elements of $\mathscr{A}(W)$; so without loss of generality we may assume that $W^{\prime}=W_{J}$ for some $J \subseteq \Pi$.

Let $V^{\prime}$ be the subspace of $V$ spanned by the root system of $\alpha\left(W_{J}\right)$, and let $v$ be a nonzero element of the orthogonal complement of $V^{\prime}$ in $V$. Then $w v=v$ for all $w \in \alpha\left(W_{J}\right)$, and in particular $\alpha\left(r_{a}\right) v=v$ for all roots $a \in \Phi_{J}^{+}$. There is at least one $a \in \Phi_{J}^{+}$such that $\alpha\left(r_{a}\right) \neq r_{a}$, since otherwise we would have $\alpha(w)=w$ for all $w \in W_{J}$. Moreover, $\alpha\left(r_{a}\right) \neq r_{a}$ implies that $\alpha\left(r_{a}\right)=r_{a} z$. Now since $z$ acts on $V$ as multiplication by -1 , if $\alpha\left(r_{a}\right)=r_{a} z$ then $r_{a} v=-v$, which implies that $v$ is a scalar multiple of $a$. Since there is at least one such $a \in \Phi_{J}^{+}$, it is unique. Fix this root $a$, and note that it is orthogonal to $V^{\prime}$.

If $b \in \Phi_{J}^{+}$and $b \neq a$ then $r_{b} a=\alpha\left(r_{b}\right) a=a$. Hence $a$ is orthogonal to $\Phi_{J}^{+} \backslash\{a\}$. Thus $r_{a}$ generates a component of $W_{J}$ of type $A_{1}$, and every other component of $W_{J}$ is contained in $\alpha\left(W_{J}\right)$. Furthermore, $r_{a}$ is not conjugate in $W$ to any other reflection $r_{b} \in W_{J}$, since $f\left(r_{a}\right)=z \neq f\left(r_{b}\right)$. So $W$ is of type $B_{n}$ or $F_{4}$ and $W_{J}$ is of type $A_{1} \times A_{n-2}$, where $n$ is the rank of $W$. Let $K=J \backslash\{a\}$, and note that $W_{K}$ is a parabolic subgroup of $\alpha\left(W_{J}\right)$. Thus $\alpha\left(W_{J}\right)$ is also of type $A_{1} \times A_{n-2}$. If $n>3$ then the centre of $\alpha\left(W_{J}\right)$ has order two and is generated by a reflection; however, this contradicts the fact that $r_{a} z$ is not a reflection, since it acts as multiplication by -1 on the space $V^{\prime}$, which has dimension greater than 1 . So $n=3$, and $W$ is of type $B_{3}$. Writing $b$ for the unique element of $J \backslash\{a\}$ we find that the two reflections in $\alpha\left(W_{J}\right)$ are $r_{b}$ and $r_{b} r_{a} z$. But these are conjugate in $W$, whereas in type $B_{3}$ the parabolic subgroups of type $A_{1} \times A_{1}$ are generated by a pair of non-conjugate reflections.

Proposition 34. Let $W$ be a finite irreducible Coxeter group of rank $n$, and suppose that $W$ is not of type $A_{5}$. Let $\alpha \in A u t(W)$, and suppose that for every reflection $r \in W$ the element $\alpha(r)$ lies in a parabolic subgroup of $W$ of rank less than $n-1$. Then $\alpha \in R(W)$.

Proof. Suppose that $\alpha \notin R(W)$. The image of a proper parabolic subgroup under the action of an element of $R(W)$ is clearly always a proper parabolic subgroup. So we may replace $\alpha$ by $\alpha \beta$ for any $\beta \in R(W)$ without affecting either the hypotheses of the proposition or the assumption that $\alpha \notin R(W)$. Since $W$ is not of type $A_{5}$ we may assume that $\alpha \in \mathscr{A}(W)$. Let $\alpha=\alpha_{f}$, where $f \in \mathscr{H}$. Now $f \neq 1$ since $\alpha \neq 1$, and so there exists a simple reflection $r_{a}$ such that $f\left(r_{a}\right)=z$. It follows that $r_{a} z=\alpha\left(r_{a}\right)$ lies in a parabolic subgroup $W^{\prime}$ of rank less than $n-1$. If $V^{\prime}$ is the subspace of $V$ spanned by the root system of $W^{\prime}$ then all elements of $W^{\prime}$ act trivially on the quotient space $V / V^{\prime}$, which has dimension at least 2 . So $r_{a} z$ has 1 as a repeated eigenvalue, contradicting the fact that it acts as -1 on the $(n-1)$-dimensional space $\{v \in V \mid v \cdot a=0\}$.

Note that if $W$ is of type $A_{5}$ then the automorphisms that do not preserve reflections take them to conjugates of the central element of a parabolic subgroup of type $A_{1}^{3}$. However, to deal with type $A_{5}$ we have the following fact.

Proposition 35. Let $W$ be a Coxeter group of type $A_{5}$, and let $\alpha \in A u t(W)$. If there exists a nontrivial proper parabolic subgroup $W^{\prime}$ of $W$ such that $\alpha\left(W^{\prime}\right)$ is also a parabolic subgroup of $W$ then $\alpha$ is inner.

Proof. We can identify $W$ with the symmetric group of degree 6, and, modifying $\alpha$ by an appropriate inner automorphism, we may assume that the action of $\alpha$ on the generators 
$r_{i}=(i, i+1)($ for $1 \leq i \leq 5)$ is as follows:

$$
\begin{aligned}
& (12) \mapsto(13)(24)(56) \\
& (23) \mapsto(16)(25)(34) \\
& (34) \mapsto(14)(23)(56) \\
& (45) \mapsto(16)(24)(35) \\
& (56) \mapsto(12)(34)(56) .
\end{aligned}
$$

If $W^{\prime}$ is a nontrivial proper parabolic subgroup such that $\alpha\left(W^{\prime}\right)$ is also parabolic then $\alpha\left(W^{\prime}\right)$ certainly contains an element from the conjugacy class of $W$ containing the element (12)(34)(56). Hence $\alpha\left(W^{\prime}\right)$ is of type $A_{1}^{3}$ or $A_{1} \times A_{3}$. Since $\alpha^{2}$ is inner, it suffices now to check that neither $\alpha\left(\left\langle r_{1}, r_{3}, r_{5}\right\rangle\right)$ nor $\alpha\left(\left\langle r_{1}, r_{2}, r_{3}, r_{5}\right\rangle\right)$ is a parabolic subgroup. We leave this straightforward task to the reader.

Suppose that $W$ is a nearly finite Coxeter group of rank $n$ with no infinite edge labels. Suppose that $a \in \Pi$ is such that $J=\Pi \backslash\{a\}$ is irreducible and of finite type, and let $\alpha$ be an automorphism of $W$. From Corollary 12 we know that $\alpha\left(W_{J}\right)$ is a maximal finite parabolic subgroup. Replacing $\alpha$ by its composite with an inner automorphism permits us to assume that $\alpha\left(W_{J}\right)=W_{K}$ for some $K \subseteq \Pi$. Clearly the rank of $W_{K}$ is at most $n-1$.

We claim that $W_{K}$ is of the same type as $W_{J}$. This depends on the following fact, whose proof we omit.

Proposition 36. Suppose that $W$ is an irreducible finite Coxeter group that is abstractly isomorphic to a direct product of two nontrivial Coxeter groups. Then $W$ is either of type $B_{k}$ for some odd $k>1$ or $I_{2}(2 m)$ for some odd $m>1$. The factors are of types $A_{1}$ and $D_{k}$ in the former case (or $A_{1}$ and $A_{3}$ if $k=3$ ), and of types $A_{1}$ and $I_{2}(m)$ in the latter case.

This can be proved, for example, by an examination of the list of normal subgroups of finite irreducible Coxeter groups given by Maxwell [13]. The proposition tells us that if an irreducible finite Coxeter group is abstractly isomorphic to a reducible Coxeter group, then the rank of the reducible group is one greater than the rank of the irreducible group.

Hence in our situation above, $W_{K}$ must be irreducible. As we noted in the proof of Proposition 25, if two irreducible finite Coxeter groups are abstractly isomorphic then they are of the same type. So $W_{J}$ and $W_{K}$ are of the same type. Thus we have proved the following result.

Theorem 37. If $W$ is a nearly finite Coxeter group of rank $n$, and $W_{J}$ a standard parabolic subgroup of $W$ that is irreducible and of rank $n-1$, then any automorphism of $W$ will map $W_{J}$ to a conjugate of a standard parabolic subgroup $W_{K}$ of the same type as $W_{J}$.

The following is Lemma 9 in [9].

Lemma 38. If $W$ is any infinite irreducible Coxeter group then the only graph automorphism that is inner is the identity.

Our objective is to prove the following result.

Theorem 39. Suppose that $W$ is a nearly finite Coxeter group with finite edge labels, and suppose that $J=\Pi \backslash\{a\}$ is irreducible and of finite type. Suppose also that $m_{a b}$ is odd for at least one $b \in J$. Then all automorphisms of $W$ are inner by graph, and indeed

$$
\operatorname{Aut}(W)=\operatorname{Inn}(W) \rtimes \operatorname{Gr}(W),
$$

where $\mathrm{Gr}(W)$ is the group of all graph automorphisms of $W$.

Proof. Since Lemma 38 above tells us that $\operatorname{Inn}(W) \cap \operatorname{Gr}(W)=\{1\}$, the assertion that $\operatorname{Aut}(W)=\operatorname{Inn}(W) \rtimes \operatorname{Gr}(W)$ will follow once it has been shown that all automorphisms are inner by graph. By Theorem 29 it suffices to prove that all automorphisms preserve reflections. 
Suppose, for a contradiction, that $\alpha \in \operatorname{Aut}(W)$ does not preserve reflections. By Theorem 37 we may assume that $\alpha\left(W_{J}\right)=W_{K}$ for some $K \subseteq \Pi$ of the same type as $J$. Let $\beta: W_{K} \rightarrow W_{J}$ be an isomorphism that takes simple reflections to simple reflections. Since $r_{a}$ is conjugate to an element of $W_{J}$ there exists at least one reflection $r \in W_{J}$ such that $\alpha(r)$ is not a reflection; hence the automorphism $\gamma$ of $W_{J}$ given by $w \mapsto \beta(\alpha(w))$ is not in $R\left(W_{J}\right)$.

Let us first assume that $J$ and $K$ are not of type $A_{5}$. By Proposition 34 there is a $b \in J$ such that $\gamma(b)$ does not lie in any parabolic subgroup of $W_{J}$ of rank less than $n-2$, where $n$ is the rank of $W$. So $\alpha\left(r_{b}\right)$ does not lie in any parabolic subgroup of $W_{K}$ of rank less than $n-2$. Since $m_{a b} \neq \infty$ there exists at least one maximal finite parabolic subgroup $W^{\prime}$ containing both $r_{a}$ and $r_{b}$. Suppose there are more than one of these, say $W^{\prime}$ and $W^{\prime \prime}$. Then $\alpha\left(W^{\prime}\right)$ and $\alpha\left(W^{\prime \prime}\right)$ are distinct proper parabolic subgroups of $W$, and so $\alpha\left(W^{\prime}\right) \cap \alpha\left(W^{\prime \prime}\right)$ is a parabolic subgroup of $W$ of rank at most $n-2$ (by Lemma 7 ). Furthermore, $\alpha\left(W^{\prime}\right) \cap \alpha\left(W^{\prime \prime}\right)$ is not contained in $W_{K}$, since $W^{\prime} \cap W^{\prime \prime}$ is not contained in $W_{J}$. So $\alpha\left(W^{\prime}\right) \cap \alpha\left(W^{\prime \prime}\right) \cap W_{K}$ has rank at most $n-3$ and is a parabolic subgroup of $W_{K}$ containing $\alpha\left(r_{b}\right)$. This contradiction shows that there is a unique maximal finite parabolic subgroup $W^{\prime}$ containing $r_{a}$ and $r_{b}$. Since there is obviously a maximal finite standard parabolic subgroup containing $r_{a}$ and $r_{b}$, it follows from Lemma 12 that $W^{\prime}=W_{L}$ for some $L \subseteq \Pi$. If $W_{L}$ has rank $n-2$ or less then $\alpha\left(W_{L}\right) \cap W_{K}$ has rank $n-3$ or less and is a parabolic subgroup of $W_{K}$ containing $r_{b}$; as before, this is a contradiction. So $L=\Pi \backslash\{c\}$ for some $c \in J$; moreover, $\alpha\left(W_{L}\right) \cap W_{K}$ has rank $n-2$.

Since $W_{J}$ is not of type $A_{5}$, there is a reflection preserving automorphism $\delta$ of $W_{J}$ such that $\delta \gamma \in \mathscr{A}\left(W_{J}\right)$. Now

$$
(\delta \gamma)\left(W_{L \cap J}\right)=\delta\left(\beta\left(\alpha\left(W_{L} \cap W_{J}\right)\right)\right)=\delta\left(\beta\left(\alpha\left(W_{L}\right) \cap W_{K}\right)\right)
$$

is a maximal parabolic subgroup of $W_{J}$, by Proposition 32. So by Proposition 33 it follows that $(\delta \gamma)(w)=w$ for all $w \in W_{L \cap J}$. Since $r_{b} \in W_{J \cap L}$ and $\delta$ preserve reflections, it follows that $\gamma\left(r_{b}\right)$ is a reflection, and hence $\alpha\left(r_{b}\right)$ is a reflection. This is a contradiction, and completes the proof in the case that $J$ and $K$ are not of type $A_{5}$.

So suppose that $J$ and $K$ are of type $A_{5}$. Let $W_{L}$ be a maximal finite standard parabolic subgroup of $W$ containing $r_{a}$ and $r_{b}$. Then $\alpha\left(W_{L}\right) \cap W_{K}$ is the intersection of two maximal finite subgroups of $W$, and hence is a nontrivial proper parabolic subgroup of $W_{K}$. So $\beta\left(\alpha\left(W_{L}\right) \cap W_{K}\right)=\gamma\left(W_{L \cap J}\right)$ is a proper parabolic subgroup of $W_{J}$. By Proposition 35 it follows that $\gamma$ is inner, contradicting the fact that $\gamma \notin R\left(W_{J}\right)$.

\section{REFERENCES}

[1] N. Bourbaki, Groupes et algèbres de Lie, Chapitres 4, 5 et 6, Hermann, Paris, 1968.

[2] Brigitte Brink, The dominance minimal roots, Journal of Algebra 206 (1998), 371-412.

[3] R. W. Carter, Conjugacy classes in the Weyl group, Compositio Mathematica 25 (1972), 1-59.

[4] Roger W. Carter, Finite Groups of Lie Type: Conjugacy Classes and Complex Characters., J. Wiley \& Sons, 1985

[5] R. Charney and M. Davis, When is a Coxeter system determined by its Coxeter group? J. London Math. Soc. 61 (2000), 441-461.

[6] V. V. Deodhar, On the root system of a Coxeter group, Communications in Algebra 10 (1982), 611-630.

[7] W. N. Franzsen, Automorphisms of rank three Coxeter groups with infinite bonds, Journal of Algebra 248 (2002), 381-396

[8] — Automorphisms of Coxeter Groups, PhD thesis, January 2001 (University of Sydney).

[9] W. N. Franzsen and R. B. Howlett, Automorphisms of Coxeter groups of rank three, Proceedings of the American Mathematical Society 129 (2001), 2607-2616.

[10] R. B. Howlett, P. J. Rowley, and D. E. Taylor, On outer automorphism groups of Coxeter groups, Manuscripta Mathematica 93 (1997), 499-513.

[11] James E. Humphreys, Reflection Groups and Coxeter Groups, Cambridge University Press, 1990 (Cambridge Studies in Advanced Mathematics, Vol. 29).

[12] L. D. James, Complexes and Coxeter groups-operations and outer automorphisms, Journal of Algebra 113 (1988), 339-345.

[13] G. Maxwell, The normal subgroups of finite and affine Coxeter groups, Proceedings of the London Mathematical Society 3 (1998), 359-382. 
[14] B. Mühlherr, Automorphisms of graph-universal Coxeter groups, Journal of Algebra 200 (1998), 629-649.

[15] R. W. Richardson, Conjugacy classes of involutions in Coxeter groups, Bulletin of the Australian Mathematical Society 26 (1982), 1-15.

[16] J. Tits, Sur le groupe des automorphismes de certain groupes de Coxeter, Journal of Algebra 113 (1988), $346-357$.

Australian Catholic University, 25A Barker Rd, StrathField NSW 2135, Australia

E-mail address: b.franzsen@mary.acu.edu.au

School of Mathematics and Statistics, University of Sydney, NSW 2006, Australia

E-mail address: R.Howlett@maths.usyd.edu.au 